\title{
Slat Noise Trend Predictions using CAA with Stochastic Sound Sources from a Random Particle Mesh method (RPM)
}

\author{
R. Ewert* \\ DLR, Institute of Aerodynamics and Flow Technology \\ Technical Acoustics, Lilienthalplatz 7, 38108 Braunschweig, Germany \\ e-mail: roland.ewert@dlr.de
}

\begin{abstract}
Slat Noise simulations are carried out for a high-lift airfoil. The high-lift airfoil geometry is a two-element slat-airfoil configuration without deployed flap in order to avoid additional sound sources at the flap. A low-cost CAA approach is applied, which is based on acoustic perturbation equations (APE) in the time-domain that are forced by stochastic sound sources. The stochastic model was introduced in AIAA 2005-2862 and is based on the spatial convolution of white-noise with a filter kernel and can reproduce target distributions of turbulent kinetic energy and length scales, e.g., provided by a steady RANS computation of the turbulent flow problem. The Mach number scaling law of the broadband slat noise component is evaluated based on three different freestream velocities $(M=0.088,0.118$, 0.165). The capability of the stochastic method to reproduce a target turbulence kinetic energy topology is studied. The effect of model parameter variations on the quality of the reproduced turbulent kinetic energy distribution and its effect on the predicted far-field spectrum is evaluated. Only a small dependence on the model parameter is found. The effect of slat gap variation on the broadband acoustic far-field is studied for three different slat configurations, which encompass beside the reference slat position one configuration with reduced, and one with increased slat gap. Previous experimental findings at a full three-element high-lift airfoil indicated an effective dependence of the slat gap width on the acoustic far-field with typically $5 \mathrm{~dB}$ reduction for a slat gap reduction of about $15 \%$ due to a decrease of the velocity of about $25 \%$. The current study investigates this effect for the two-element high-lift configuration.
\end{abstract}

\section{Introduction}

ARCRAFT noise reduction as achieved through the development and application of high bypass low noise Aturbofan engines has shifted the focus of interest to airframe noise as an equally important noise source during the approach phase. Experimental studies have identified deployed slats as prominent noise contributors. ${ }^{1,2}$ To reduce noise levels further, numerical tools will become necessary in the future to achieve an optimized low noise design of airframe components. Therefore, the development of cheap and quick computational methods, which can be used in an optimization process, is essential. Over recent years computational methods have been improved for computational aeroacoustics (CAA) purposes (in particular: high-order non-dispersive spatial and temporal discretization schemes and high-quality non-reflecting boundary conditions have been introduced). A key point, however, is how an acoustic solver based on the linearized Euler

*Research Engineer, DLR, Institute of Aeordynamics and Flow Technology, Technical Acoustics, Braunschweig, Germany, AIAA Member. 
equations (LEE) or derivatives of it can be used for designing low-noise airframe components (design-to-noise approach). A presumably accurate noise prediction methodology arises if appropriate acoustic sources of the propagation equations are computed from time accurate LES or DNS simulations of the turbulent near field. However, such approaches are too time consuming to be used in a design process that requires numerous evaluations of design modifications.

In this paper the application of a cheaper computational approach to a slat noise problem is studied. It involves CAA techniques in conjunction with stochastic sound sources in the time domain ('low-cost

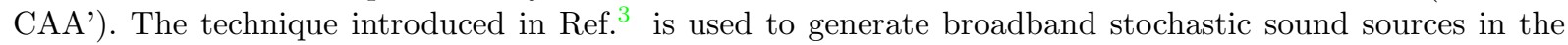
time-domain. It is based on the spatial filtering of white-noise and is capable to reproduce exactly the second-order two-point correlation tensor of homogeneous isotropic turbulence in two or three dimensions. The stochastic method is time- and memory efficient, strictly solenoidal, and can be easily applied to highly non-uniform mean-flow fields (e.g. as apparent in the slat-cove region of a high-lift airfoil) using local results for the kinetic turbulent energy and the corresponding turbulent length scale from a RANS solution of the turbulent mean-flow. Furthermore, it avoids the occurrence of shear decorrelations and resolves broadband spectra continously. A further extension to non-homogeneous anisotropic solenoidal flows is possible with the transformation proposed by Smirnov et al. ${ }^{4}$

The slat noise simulations are carried out for a modified two-element high-lift configuration, which consists of a slat and a main-element. The slat geometry and the main-element correspond up to $40 \%$ chord length to the geometry of a reference three-element high-lift configuration. The remaining part of the main-element is modified by increasing the camber to aerodynamically compensate for the omitted flap. The geometrical design was developed in the German project Frequenz that aims at studying design modifications at three selected aeroacoustic problems numerically and experimentally. A removed flap was chosen to avoid additional flap sound sources in the experiments that accompany the numerical simulations. The additional flap sources would otherwise reduce the meaningful signal-to-noise ratio in acoustic far-field measurements.

This paper is organized as follows. Section II discusses some fundamental features of the stochastic modeling of turbulent velocity fields that is applied in this work. Since fluctuating velocity components are deduced from a fluctuating streamfunction, the statistical features that have to met by the fluctuating streamfunction are identified. Section III discusses the general procedure how to set-up a fluctuating streamfunction with previously identified statistical characteristics, and Section IV presents details about the numerical discretization of this procedure. Acoustic simulation techniques are are introduced in Section V. Section VI discusses the computational results for the two-element airfoil configuration. Finally, conclusions are drawn in Section VII.

\section{Stochastic sound source modeling}

Sound generation and propagation due to turbulent sources is described by acoustic analogies, which have the form of a forced linear acoustic wave equation with wave operator $\mathcal{L}$, acoustic variable $p^{\prime}$, and source $Q$

$$
\mathcal{L} p^{\prime}(\boldsymbol{x}, t)=Q(\boldsymbol{x}, \boldsymbol{u}, t) .
$$

Starting from eq. (1) the far-field spectral density of the acoustic variable can be expressed in terms of the statistical properties of the source ${ }^{5}$

$$
S(\boldsymbol{x}, \omega)=\int_{\boldsymbol{y}} \int_{\boldsymbol{r}} \widetilde{G^{*}}(\boldsymbol{x}, \boldsymbol{y}, \omega) \widetilde{G}(\boldsymbol{x}, \boldsymbol{y}+\boldsymbol{r}, \omega) \widetilde{Q}_{12}(\boldsymbol{y}, \boldsymbol{r}, \omega) \mathrm{d} \boldsymbol{r} \mathrm{d} \boldsymbol{y} .
$$

Here $S(\boldsymbol{x}, \omega)$ stands for the power spectrum of the acoustic variable $p^{\prime}$ in (1), i.e.,

$$
\overline{p^{\prime 2}}=\frac{1}{2 \pi} \int_{-\infty}^{\infty} S(\boldsymbol{x}, \omega) \mathrm{d} \omega
$$

The quantities $\widetilde{G}$ and $\widetilde{G^{*}}$ denote respectively the exact Green's function and its conjugate complex in the frequency domain. They are defined by the linear wave-operator of eq. (1) and the appropriate boundary 
conditions. Furthermore, $\widetilde{Q}_{12}$ is a cross spectral density which is obtained by Fourier transform of the twopoint space-time correlation of source $Q$ in eq. (1) between source points $\boldsymbol{y}_{1}=\boldsymbol{y}$ and $\boldsymbol{y}_{2}=\boldsymbol{y}+\boldsymbol{r}$ and time separation $\tau=t_{1}-t_{2}$

$$
\begin{gathered}
\widetilde{Q}_{12}(\boldsymbol{y}, \boldsymbol{r}, \omega)=\int_{-\infty}^{\infty} R(\boldsymbol{y}, \boldsymbol{r}, \tau) e^{i \omega \tau} \mathrm{d} \tau, \\
R(\boldsymbol{y}, \boldsymbol{r}, \tau)=\overline{Q(\boldsymbol{y}, t) Q(\boldsymbol{y}+\boldsymbol{r}, t+\tau)} .
\end{gathered}
$$

Eq. (2) is the basis of all statistical noise theories in the frequency domain. It evidences that the far-field spectrum is completely determined if just the two-point space-time correlation of the sources (eq. (4)) is known. For turbulence related noise sources this means a reduction in the amount of necessary statistical information, since the underlying turbulent flow field is determined by an infinite set of m-point nth-order correlations. Note that the acoustic far-field spectrum is the only quantity that in principle has to be known for acoustic purposes. Other quantities like directivities and root-mean square values can be deduced from it.

A synthetic fluctuating source $\mathrm{Q}(\boldsymbol{x}, \boldsymbol{u}, \mathrm{t})$ which realizes just the proper two-point space-time correlations, eq. (4), will yield a far-field spectrum that satisfies eq. (2) when inserted into the time-domain analogy eq. (1). In other words, a synthetic fluctuating source term that realizes the proper two-point space-time correlations gives an alternative way to solve eq. (2). It yields an exact solution to the acoustic far-field spectrum if the underlying second-order two-point correlations are realized through the fluctuating source. These correlation might be determined by means of measurements or from steady RANS solutions of the turbulent flow problem.

As discussed by Tam and Auriault, ${ }^{6}$ one appropriate model space time-correlation function characterized by three parameters may be expressed by Gaussian spatial and exponential temporal correlations. It closely approximates the measurements of Davies et al..$^{7}$ for the fluctuating axial velocity component in jets. This generic (normalized) two-point space-time correlation can be written as

$$
\mathcal{R}(\boldsymbol{y}, \boldsymbol{r}, \tau)=\exp \left\{-\frac{\tau}{\tau_{\mathrm{s}}(\boldsymbol{y})}-\frac{\pi\left(\boldsymbol{r}-\boldsymbol{u}_{c}(\boldsymbol{y}) \tau\right)^{2}}{4 l_{\mathrm{s}}(\boldsymbol{y})^{2}}\right\}
$$

Parameters $\tau_{s}$ and $l_{\mathrm{s}}$ define respectively the correlation time- and length scales and depend in general, as indicated, on position. Taylor's hypothesis is taken into account by the convection velocity $\boldsymbol{u}_{c}$. In a comoving frame of reference, just considering frozen convection through $\tau_{\mathrm{s}} \rightarrow \infty$, the correlation takes on a Gaussian form. Although eq. (5) is only an approximation of the velocity correlations, it is used with great success to model the broadband (fine scale turbulence) features of the full jet noise source terms, ${ }^{6,8}$ which are in general non-linear functions of the velocities. Tam and Auriault solve problem (2), with sources expressed through eq. (5), using a CAA method to compute the flow Green's function in eq. (2). It applies the adjoint Euler equations in a reciprocal approach. Agarwal and Morris recently extended the statistical source description through eq. (5) to an airframe noise problem, ${ }^{9}$ exploiting also acoustic reciprocity.

The new stochastic method, which was introduced in Ref., ${ }^{3}$ is capable of generating spatially and temporally fluctuating quantities that reproduce target two-point space-time correlations of the type described by eq. (5), whereby local target values for $\tau_{\mathrm{s}}, \boldsymbol{u}_{c}$, and $l_{\mathrm{s}}$ can be realized. A direct approach to solve eq. (2) via eq. (1) with appropriately modeled fluctuating source has the advantage that only one computation is necessary to determine the solution for all frequency bands and observer positions, whereas an adjoint approach in the frequency domain needs for $n$ observer positions and $m$ frequency bands $n \times m$ computations.

Note that the source $Q(\boldsymbol{x}, \boldsymbol{u}, t)$ in eq. (1) usually is a function of the turbulent velocity fluctuations $\boldsymbol{u}$ if vortex sound is considered. If not the statistical properties of the source (described by eq. 4) have to be modeled but rather the underlying characteristics of the turbulent velocity fluctuations $\boldsymbol{u}$, some additional physical features may have to be included into the fluctuation model. For instance, velocity fluctuations originating from vorticity are divergence-free (solenoidal). Deviations of the modeled fluctuations from this constraint may give rise to spurious sound sources. The divergence-free condition for the velocities can 
be satisfied by defining the fluctuating quantity, which is resolved by the stochastic model, to be a scalar streamfunction $\psi(\boldsymbol{x}, t)$ (not e.g. the source $Q$ ), from which velocity components are deduced subsequently. Hence, in $2 \mathrm{D}$ the velocities read

$$
u^{\prime}=\frac{\partial \psi}{\partial y}, \quad v^{\prime}=-\frac{\partial \psi}{\partial x},
$$

and are strictly solenoidal. However, the differentiations with respect to one spatial direction, which appear in eq. (6), causes a reduction of the integral length-scale of the velocity fluctuations in that particular direction (due to the amplification of higher wave-numbers in wave-number space). Hence, a fluctuation model that realizes for the fluctuating streamfunction $\psi(\boldsymbol{x}, t)$ (isotropic) correlations of the type described by eq. (5) will cause an anisotropic distortion of the resulting velocity field. It is worth to note and to show that these distorted velocity fields exhibit rather physical characteristics.

Starting point to show this feature is to consider the correlation of the fluctuating scalar streamfunction,

$$
\mathcal{R}(r, \tau)=\overline{\psi\left(\boldsymbol{r}_{1}, t_{1}\right) \psi\left(\boldsymbol{r}_{2}, t_{2}\right)},
$$

to have the formal shape defined by eq. (5), where $r=\left|\boldsymbol{r}_{1}-\boldsymbol{r}_{2}\right|$ and $\tau=\left|t_{1}-t_{2}\right|$.

As was shown by Careta et al. ${ }^{10}$ a scalar streamfunction with isotropic correlation accomplishes a perfect description of isotropic homogeneous turbulence in $2 \mathrm{D}$ in terms of the two-point correlations. Homogeneity and isotropy requirements imply that the two-point two-time correlation tensor $\mathcal{R}_{i j}(r, \tau)$,

$$
\mathcal{R}_{i j}(r, \tau)=\overline{u_{i}\left(\boldsymbol{r}_{1}, t_{1}\right) u_{j}\left(\boldsymbol{r}_{2}, t_{2}\right)},
$$

has to be written in terms of radial functions $f(r)$ and $g(r)$ as

$$
\mathcal{R}_{i j}(r, 0)=[f(r)-g(r)] n_{i} n_{j}+g(r) \delta_{i j}
$$

The divergence-free condition connects the longitudinal correlation function $f(r)$ with the lateral correlation function $g(r)$ in $2 \mathrm{D}$ via

$$
g(r)=f(r)+r \frac{\mathrm{d} f(r)}{\mathrm{d} r}
$$

In $3 \mathrm{D}$ this relation becomes ${ }^{11}$

$$
g(r)=f(r)+\frac{r}{2} \frac{\mathrm{d} f(r)}{\mathrm{d} r} .
$$

Straightforward algebra shows that the properties (9) and (10) are satisfied for a fluctuating scalar streamfunction after identifying $f(r)$ and $g(r)$ in terms of the scalar field $\mathcal{C}(r):=\mathcal{R}(r, 0)$ as

$$
f(r)=-\frac{\mathcal{C}^{\prime}(r)}{r}, \quad g(r)=-\mathcal{C}^{\prime \prime}(r)
$$

where $\mathcal{C}^{n^{\prime}}(r)=\mathrm{d}^{n} \mathcal{C}(r) / \mathrm{d} r^{n}$. It is easy to prove that eq. (10) is satisfied when inserting expressions (12) into it. Hence, the only constraint to perfectly realize the correlation tensor of homogeneous isotropic turbulence (HIT) in 2D is that the correlation of the fluctuating scalar streamfunction depends for $\tau=0$ just on radial distance. This constraint is satisfied for a fluctuating streamfunction realizing eq. (5) in 2D (with $\boldsymbol{r} \in \boldsymbol{R}^{2}$ ).

By normalizing $\mathcal{R}$ in eq. (5) with $2 l_{\mathrm{s}}^{2} / \pi$, the longitudinal correlation function can be deduced from eq. (12) to be

$$
f(r)=\exp \left(-\frac{\pi}{4} \frac{r^{2}}{l_{\mathrm{s}}^{2}}\right)
$$

The related integral length scale reads

$$
\begin{aligned}
& L=\int_{0}^{\infty} f(r) \mathrm{d} r=\int_{0}^{\infty} \exp \left(-\frac{\pi}{4} \frac{r^{2}}{l_{\mathrm{s}}^{2}}\right) \mathrm{d} r=l_{\mathrm{s}} . \\
& \text { American Institute of Aeronautics and Astronautics }
\end{aligned}
$$


Hence, $l_{\mathrm{s}}$ is also the integral length scale of the longitudinal correlation function $f(r)$.

An extension of the method of Careta et al. to 3D HIT is straight forward by introducing a fluctuating vector streamfunction $\boldsymbol{\psi}(\boldsymbol{x}, t)$, which defines the fluctuating velocities via

$$
\boldsymbol{u}^{\prime}=\nabla \times \psi
$$

It is obvious that $\boldsymbol{u}^{\prime}$ satisfies the divergence-free condition. Furthermore, if the components of the vector streamfunction are statistically independent, each having similar spatial correlations $\mathcal{C}(r)=\mathcal{R}(r, 0)$, or equivalently

$$
\overline{\psi_{i} \psi_{j}}=\delta_{i j} \mathcal{C}(r)
$$

it can be shown that the velocity correlations satisfy eq. (9) in conjunction with eq. (11), hence realize the correlation tensor of HIT in 3D. Using eq. (15) straightforward algebra shows the longitudinal and lateral correlation functions are expressed through the correlation function of the streamfunction components by

$$
f(r)=-\frac{2 \mathcal{C}^{\prime}}{r}, \quad g(r)=-\mathcal{C}^{\prime \prime}-\frac{\mathcal{C}^{\prime}}{r}
$$

These expressions differ slightly from their 2D counterpart, eq. (12). However, the relation between the longitudinal and lateral correlation function in 3D is perfectly satisfied, which can be shown by inserting the expressions (16) into eq. (11). The 3D longitudinal correlation function $f(r)$ differs from the $2 \mathrm{D}$ expression only by a factor 2 , which has no influence on the normalized longitudinal correlation with $f(0)=1$. Therefore, based on a streamfunction correlation expressed through eq. (5), the (normalized) correlation function eq. (13) is still valid. Note that the additional term for $g(r)$ in eq. (16) causes the 3D lateral correlation function to have a less pronounced negative dip compared to the $2 \mathrm{D}$ case, eq. (12).

Anisotropic turbulence based on modeled 3D homogeneous isotropic turbulence can be achieved with the additional velocity transformation proposed by Smirnov et al. ${ }^{4}$ The transformation is applied to the initial homogeneous isotropic velocities of eq. (14). The outcome of the transformation is a time-dependent flowfield $v_{i}^{\prime}$ with one-point correlation functions $\overline{v_{i}^{\prime} v_{j}}$ equal to that of the local (anisotropic) Reynolds stress tensor $\tau_{i j}=\mathcal{R}_{i j}(0,0)$ and turbulent length/time scales equal to $l_{s}$ and $\tau_{s}$. The flow-field is also exactly divergence free for homogeneous turbulence and to a high degree divergence-free for inhomogeneous turbulence.

In this paper the method is applied for convenience and as a first feasibility study not in full rigor to a slat-noise problem. For airframe noise problems like wing slat- or trailing edge noise, only the vorticity vector component in spanwise direction will contribute to the sound generation. The velocity field related to this single vorticity component can be resolved by one scalar streamfunction. If taken into account, anisotropy would appear through a local factor that determines the auto-correlation of the spanwise vorticity component with respect to the trace of all vorticity correlations. Neglecting anisotropy tacitly means using a constant anisotropy factor of $1 / 3$. We believe that anisotropy effects are of minor importance if not the absolute sound pressure levels are of interest but rather differences of levels resulting from design variations are studied. It is esteemed that the basic noise generating mechanism at a slat is the interaction of vortical disturbances with geometrical inhomogeneities. Hence, the temporal correlation (which is responsible for turbulent selfnoise generation) is neglected for the slat-noise problem by just considering frozen turbulence with $\tau_{\mathrm{s}} \rightarrow \infty$. Consequently, 2D CAA simulations are carried out in this paper with a source just realized through one scalar fluctuating streamfunction. One has to bear in mind that an additional 2D to 3D correction has to be applied to the $2 \mathrm{D}$ acoustic field. ${ }^{12}$ Except for a level off-set, which is deemed constant for all flow parameter variations, the 2D approach can be used to determine the shape of acoustic spectra, directivities, Mach number scaling laws, and the influence of design parameter variations.

\section{Stochastic fluctuating fields via spatial white-noise filtering}

This section discusses the realization of a fluctuating streamfunction $\psi(\boldsymbol{x}, t)$ whose correlations satisfy eq. (7). Spatiotemporal scales, local energy, and the convection of coherent structures are the essential 
physical features provided by two-point space-time correlations of turbulent motion as evidenced by eq. (5). Hence, one important feature must be the inclusion of vortex convection in non-uniform flow as encoded in the vorticity equation. For aeroacoustic noise mechanism based on the interaction of vorticity with solid surfaces, which is for instance responsible for trailing edge noise, a correct convection velocity is essential to achieve a physically meaningful Mach number scaling law.

A fluctuating streamfunction which satisfies all these constraints is generated by spatially filtering a white-noise field. The procedure is the discrete realization of the convolution or filtering integral

$$
\psi(\boldsymbol{x}, t)=\iint_{V_{S}} \hat{A}\left(\boldsymbol{x}^{\prime}\right) G^{0}\left(\left|\boldsymbol{x}-\boldsymbol{x}^{\prime}\right|, l_{\mathrm{s}}\left(\boldsymbol{x}^{\prime}\right)\right) \mathcal{U}\left(\boldsymbol{x}^{\prime}\right) \mathrm{d} \boldsymbol{x}^{\prime} .
$$

In eq. (17) $G^{0}$ is a filter kernel, $\mathcal{U}$ denotes a spatiotemporal white-noise field with unity spectrum, and $\psi$ is the fluctuating streamfunction, which determines the pseudo-turbulent velocity fluctuations via eq. (6). The filter kernel is normalized such that $\overline{\psi(\boldsymbol{x}, t) \psi(\boldsymbol{x}, t)}=1$ for $\hat{A}=1$. The integration is carried out on a source patch $V_{S}$. The argument of the filter kernel indicate that it is a function of the separation distance $\left|\boldsymbol{x}-\boldsymbol{x}^{\prime}\right|$, and of the locally $\boldsymbol{x}^{\prime}$-dependent kernel width $l_{\mathrm{s}}$ and kernel peak amplitude $\hat{A}$. For the simulation of frozen turbulence considered in this work, the spatiotemporal white-noise field is defined to have the properties

$$
\begin{aligned}
\overline{\mathcal{U}(\boldsymbol{x}, t) \mathcal{U}(\boldsymbol{x}+\Delta \boldsymbol{x}, t)} & =\delta(\Delta \boldsymbol{x}), \\
\frac{D_{0} \mathcal{U}}{D t} & =0,
\end{aligned}
$$

where $\delta(\Delta \boldsymbol{x})$ denotes a multi-dimensional Dirac $\delta$-function, which reads in $2 \mathrm{D} \delta(\Delta \boldsymbol{x})=\delta(\Delta x) \delta(\Delta y)$. Eq. (19) is a convection equation for the white-noise field, which describes passive convection in the mean-flow $\boldsymbol{u}_{0}$. It is to be understood such that in a locally comoving frame of reference the spatiotemporal white-noise field remains static. This condition can be satisfied even for an ideal white-noise realization (which is nondifferentiable) although the substantial time derivative $D_{0} / D t=\partial / \partial t+\boldsymbol{u}_{0} \boldsymbol{\nabla}$ involves spatial and temporal derivatives.

The method was sketched in $\operatorname{Ref}^{3}$ for a homogeneous one-dimensional problem. In this case the fluctuating field $\psi$ results from the one-dimensional convolution of the unity white-noise field $\mathcal{U}$ with a filter $\operatorname{kernel} G(r)$ :

$$
\psi(x, t)=\int_{-\infty}^{\infty} G\left(x-x^{\prime}\right) \cdot \mathcal{U}\left(x^{\prime}, t\right) \mathrm{d} x^{\prime}
$$

It was shown that a spatial Gaussian kernel

$$
G^{0}(x)=\exp \left(-\frac{\pi}{2} \frac{x^{2}}{l_{\mathrm{s}}^{2}}\right)
$$

yields for the correlation again a Gaussian function, but whose width is a factor $\sqrt{2}$ larger, i.e.

$$
\mathcal{R}^{0}(x)=\exp \left(-\frac{\pi}{4} \frac{x^{2}}{l_{\mathrm{s}}^{2}}\right)
$$

The extension of the scalar streamfunction approach to two or three dimensional source domain is straightforward. Integral (20) becomes for a $n$-dimensional problem

$$
\psi(\boldsymbol{x}, t)=\underbrace{\int \cdots \int}_{n \times} \hat{A} G^{0}\left(\boldsymbol{x}-\boldsymbol{x}^{\prime}\right) \mathcal{U}\left(\boldsymbol{x}^{\prime}, t\right) \mathrm{d} \boldsymbol{x}^{\prime} .
$$

Due to the white-noise field definition eq. (18) the normalized correlation becomes

$$
\mathcal{R}^{0}(\boldsymbol{r}, 0)=\int \cdots \int G^{0}(\boldsymbol{\xi}-\boldsymbol{r}) G^{0}(\boldsymbol{\xi}) \mathrm{d} \boldsymbol{\xi} .
$$

$$
6 \text { of } 33
$$


Since the Gaussian filter is separable, i.e. for the $n$-dimensional problem it can be split according to

$$
G^{0}(|\boldsymbol{x}|)=\prod_{i=1}^{n} G^{0}\left(x_{i}\right),
$$

the right-hand side of eq. (24) decomposes into $n$ products of decoupled one-dimensional convolutions, eventually leading with the one-dimensional findings eqs. $(21,22)$ to a correlation

$$
\mathcal{R}^{0}(\boldsymbol{x})=\exp \left(-\frac{\pi}{4} \frac{|\boldsymbol{x}|^{2}}{l_{\mathrm{s}}^{2}}\right)
$$

based on the filter kernel

$$
G^{0}(\boldsymbol{x})=\exp \left(-\frac{\pi}{2} \frac{|\boldsymbol{x}|^{2}}{l_{\mathrm{s}}^{2}}\right) .
$$

The separation property of a Gaussian filter kernel allows to carry out the integration in eq. (23) in a sequence of one-dimensional filter operations, which makes the filtering procedure very efficient.

Assuming a constant mean-flow $\boldsymbol{u}_{0}$ in (19), the white-noise property (18) becomes for $\tau \neq 0$

$$
\overline{\mathcal{U}(\boldsymbol{x}, t) \mathcal{U}(\boldsymbol{x}+\Delta \boldsymbol{x}, t+\tau)}=\delta\left(\Delta \boldsymbol{x}-\boldsymbol{u}_{0} \tau\right),
$$

which subsequently yields a normalized spatial correlation of the form

$$
\mathcal{R}^{0}(\boldsymbol{x}, \tau)=\exp \left\{-\frac{\pi\left(\boldsymbol{x}-\boldsymbol{u}_{0} \tau\right)^{2}}{4 l_{\mathrm{s}}^{2}}\right\} .
$$

Note that eq. (5) takes on the form realized through eq. (27) for $\boldsymbol{u}_{c}=\boldsymbol{u}_{0}$ and $\tau_{\mathrm{s}} \rightarrow \infty$, i.e., for frozen turbulence. To introduce an additional exponential temporal correlation as in (5) the homogeneous convection equation (19) has to be modified into a Langevin equation by adding a white-noise source term to the right-hand side, whose prefactor determines the correlation time $\tau_{\mathrm{s}}$.

A 3D extension of the streamfunction approach is possible by determining the components of the 3D vector streamfunction $\boldsymbol{\psi}$ through

$$
\psi_{i}(\boldsymbol{x}, t)=\iiint_{V_{S}} \hat{A} G^{0}\left(\boldsymbol{x}-\boldsymbol{x}^{\prime}\right) \mathcal{U}_{i}\left(\boldsymbol{x}^{\prime}\right) \mathrm{d} \boldsymbol{x}^{\prime} .
$$

Here the $\mathcal{U}_{i}$ denote three independent stochastic white-noise fields of the previously explained type and $G^{0}$ is a scalar filter kernel. The scalar amplitude $\hat{A}$ controls the amplitude of the fluctuations. Due to $\overline{\mathcal{U}_{i} \mathcal{U}_{j}}=\delta_{i j}$ the condition (15) holds for the 3D realization of a fluctuating vector streamfunction so that this extended filtering procedure is capable of providing 3D HIT.

Rigorously, the derivation presented so far is restricted to non-uniform mean-flows $\boldsymbol{u}_{0}$ and a homogeneous filter kernel that realizes constant correlations and length-scales throughout the source domain. Note that for non-uniform flows the Taylor hypothesis inherent in eq. (27) is still realized for small $\tau$ (in fact, it is the first order approximation to the convection equation eq. (19)). However, the convolution of the white-noise field causes the local convection velocity to be smeared out over the kernel support such that the effective local convection velocity of the fluctuating quantity does not longer equal the local mean-flow velocity, $\boldsymbol{u}_{c} \neq \boldsymbol{u}_{0}$. The convection velocity depends on the integral length scale $l_{\mathrm{s}}$ (vortex size); for $l_{\mathrm{s}} \rightarrow 0$ it tends towards the local mean-flow velocity $\boldsymbol{u}_{0}$. This behavior is deemed to model the complex physical convection property described through the vorticity equation, which, e.g., causes eddies in a generic turbulent boundary layer to convect with typically $66 \%$ of the free stream velocity.

Local kernels that realize inhomogeneous correlations and length-scales could be also deduced. However, the variation of these stationary quantities is small compared to the turbulent length-scale itself. As indicated by eq. (17), it is assumed that the analytical findings for homogeneous filter kernels are also valid with good accuracy for length scales $l_{\mathrm{s}}\left(\boldsymbol{x}^{\prime}\right)$ and kernel amplitudes $\hat{A}\left(\boldsymbol{x}^{\prime}\right)$ not locally varying too strong. The amplitude has to be chosen such that the locally generated turbulent kinetic energy $\bar{k}=1 / 2 \mathcal{R}_{i i}$ equals the target value. The appropriate RANS based scaling will be discussed in Section A. 


\section{Numerical discretization: A Random Particle Mesh (RPM) method}

On a discrete equidistant grid of spacing $\Delta x$, the continuous integral eq. (20) can be approximated with a finite sum

$$
\psi_{m}=\sum_{k=-N}^{N} G_{k} r_{k+m}
$$

which reads by rewriting the integral (20)

$$
\psi\left(x_{m}\right)=\sum_{k=-N}^{N} G(k \Delta x+\bar{\xi}) \int_{-\Delta x / 2}^{\Delta x / 2} \mathcal{U}\left(x_{m+k}+\xi\right) \mathrm{d} \xi .
$$

In expression (29) $\psi_{m}$ is the fluctuating quantity at grid point $m$ and $G_{k}=G(k \Delta x+\bar{\xi})$ with $\bar{\xi} \in$ $[-\Delta x / 2, \Delta x / 2]$ is the integral mean value of the filter kernel for an off-centered distance $k \Delta x$ and an integration interval $\Delta x$. Accordingly, quantity $r_{m}$ in (29) is a random value that follows by integrating the white-noise field over interval $\Delta x$ :

$$
r_{m}=\int_{-\Delta x / 2}^{\Delta x / 2} \mathcal{U}\left(x_{m}+\xi\right) \mathrm{d} \xi .
$$

$N$ defines the finite support of the filter. Eq. (29) takes on the form of a digital recursive filter (in the spatial domain). The only simplification introduced at this stage is the finite summation indices in eq. (29), which deliberately truncates the filter kernel to the finite support $\pm N \Delta x$. In an additional approximation the discrete Gaussian filter kernel is defined by choosing $\bar{\xi} \simeq 0$, i.e.,

$$
G_{k} \simeq \hat{A} \exp \left(-\frac{\pi}{2} \frac{k^{2}}{d^{2}}\right)
$$

where $d:=l_{\mathrm{s}} / \Delta x$ denotes the non-dimensionalized integral length scale. In this work it was found that a cut-off limit $\left|G_{k}^{0}\right| \geq 10^{-2}$, i.e. $N \approx \operatorname{int}(1.7 d)$, yields a sufficiently accurate approximation of integral eq. (20). The local integration of the white-noise field over $\Delta x$, eq. (30), yields a spectral cut-off of the white-noise spectrum for wave-lengths larger than $\Delta x$. The random values exhibit due to eq. (??) the properties $\overline{r_{m}}=0$ and

$$
\overline{r_{m} r_{k}}=\left\{\begin{array}{lll}
0 & \text { if } & m \neq k \\
\Delta x & \text { if } & m=k
\end{array}\right.
$$

The values for $r_{m}$ are obtained by loading each computational node with an appropriately scaled random number. An approximation to white-noise with a root-mean square (RMS) value normalized to one can be realized through a sequence of random numbers in the range $\pm \sqrt{3}$, generated with a constant clock rate $\Delta t$. The highest resolved frequency of this realization is linked to the seeding clock-rate $\Delta t$ through the sampling theorem, which states $f_{\max }=1 / 2 \Delta t$ (the highest frequency is a point-to-point oscillation with period $2 \Delta t$ ). Furthermore, to satisfy eq. (32) it is inferred that the amplitude of the random fluctuations must be in the range $\pm \sqrt{3 \Delta x}$.

The convection property in uniform flow $u_{0}$ is achieved for the equidistantly spaced $1 \mathrm{D}$ problem by shifting the random data one point downstream at the next higher time level and by updating the first point with new random data. Next, the time dependent fluctuating field at the new time level results from applying the filter to the updated random field. Hence, the discrete time increment becomes $\Delta t=\Delta x / u_{0}$.

To extend this one-dimensional discretization to two- or three-dimensional problems with inhomogeneous flows a bundle of mean-flow streamlines is introduced to resolve a portion of the turbulent flow region, in which vortex sound sources have to be computed. The source patch is constructed by following the paths of the mean-flow streamlines, which start along an upstream seeding line, to a user-defined downstream position. See e.g. Fig. 1(a) that shows a bundle of streamlines to resolve the slat-cove shear layer of a 
high-lift airfoil, whose initial streamlines are equidistantly distributed along the seeding line. The complete source domain is depicted in Fig. 1(b).

A constant time increment $\Delta t$ is introduced. It defines discrete points along each individual streamline according to the time a Lagrangian particle needs to reach the actual position starting from the upstream seeding line. Since the maximal time to reach the downstream border of a source patch differs from streamline to streamline, the number of discrete points also varies accordingly, see e.g. Fig. 2. The sketch furthermore highlights the area $\Delta A_{i j}$ surrounding each discrete point. A sequence of random number is assigned to each discrete point and represents the white-noise distribution for the related area. In an extension of the one-dimensional findings (32), these random values are found by integrating the convective white-noise field defined by eqs. $(18,19)$ over $\Delta A_{i j}$, i.e.,

$$
r_{i j}=\iint_{\Delta A_{i j}} \mathcal{U} \mathrm{d} \boldsymbol{x} .
$$

Using property (18), the time average of the random particle values becomes

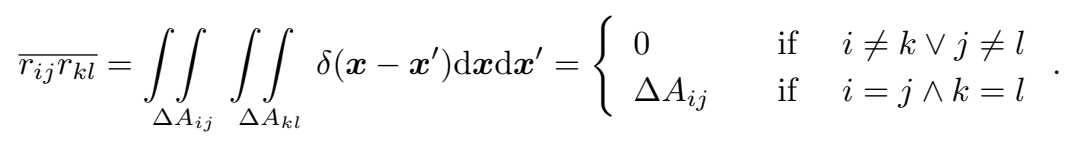

To achieve this condition through a sequence of random numbers, each element $i j$ has to take on a random value in the range $\pm \sqrt{3 \Delta A_{i j}}$.

The discretization of integral eq. (17) is achieved through a sum over all source patch elements $\Delta A_{i j}$. If $\Delta t$ agrees with the time step of the CAA method, which is used to compute the acoustic field, the complete algorithm to compute the streamfunction becomes

- for each time increment shift the unscaled random numbers (in the range $\pm \sqrt{3}$ ) one position downstream and determine local random values by scaling them with the local value of $\sqrt{\Delta A_{i j}}$

- update the first upstream position with new random values

- filter and, simultaneously, interpolate the random field onto the CAA grid

Instead of computing the contribution of all random field points to a given field position within the distance supported by the kernel, the values of the random elements are sequentially distributed into the field. Thereby the local length scale at the random point is used to scale the width of the filter kernel. Furthermore, the local value of the kinetic turbulent energy is used to scale the local amplitude $\hat{A}$ of the filter kernel. The exact value of $\hat{A}$ based on the RANS mean-flow field will be given below. The filtered values are directly computed for the relevant CAA grid points.

The filter kernel is computed in a sequence of single-dimensional filter operations, see Fig. 3a. It takes typically $15 \%$ to $1 \%$ of the time the direct evaluation of the full filter kernel would need in $2 \mathrm{D}$ and $3 \mathrm{D}$, respectively. First, the random field is filtered along the streamline for each discrete point on the streamline, using the length scale in each random point for the kernel scaling. Next, the intermediate filtered values are distributed onto the CAA grid, Fig. 3. In a preprocessing routine a base point on each streamline is identified for each CAA grid point. It marks the smallest distance to the considered grid point for all possible vectors that are normal to the streamline and run through the field point. Usually the base points lie between discrete points on the streamline. Therefore, an 4-th order interpolation is used to interpolate from the discrete points on the streamline to the base-points. The interpolated local value of the length scale at the base-point is used to compute the decay of the filter-function from the base- to the grid point. Let us denote $\Delta_{1}$ the length-scale at a given stochastic particle position $\mathbf{A}$ on the streamline, $s$ the distance along the streamline to the base-point, $\Delta_{2}$ the length-scale at the base-point and $d$ the distance to the grid point B, Fig. 3. The contribution of a random element to the grid point due to the filter kernel reads

$$
G_{A B}^{0}=G^{0}(d) G^{0}(s)=\exp \left(-\frac{\pi}{2} \frac{s^{2}}{\Delta_{1}^{2}}\right) \exp \left(-\frac{\pi}{2} \frac{d^{2}}{\Delta_{2}^{2}}\right) .
$$




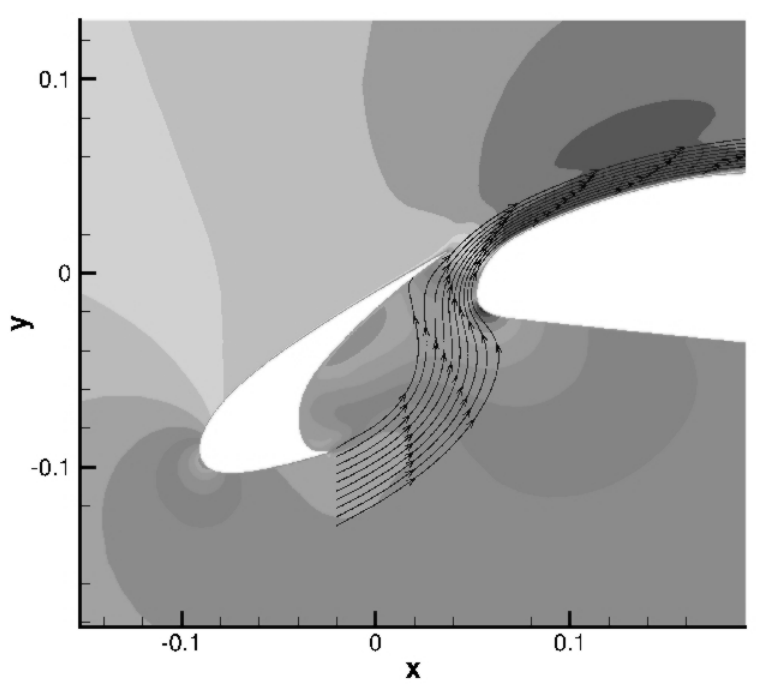

(a) Streamlines in the slat-cove.

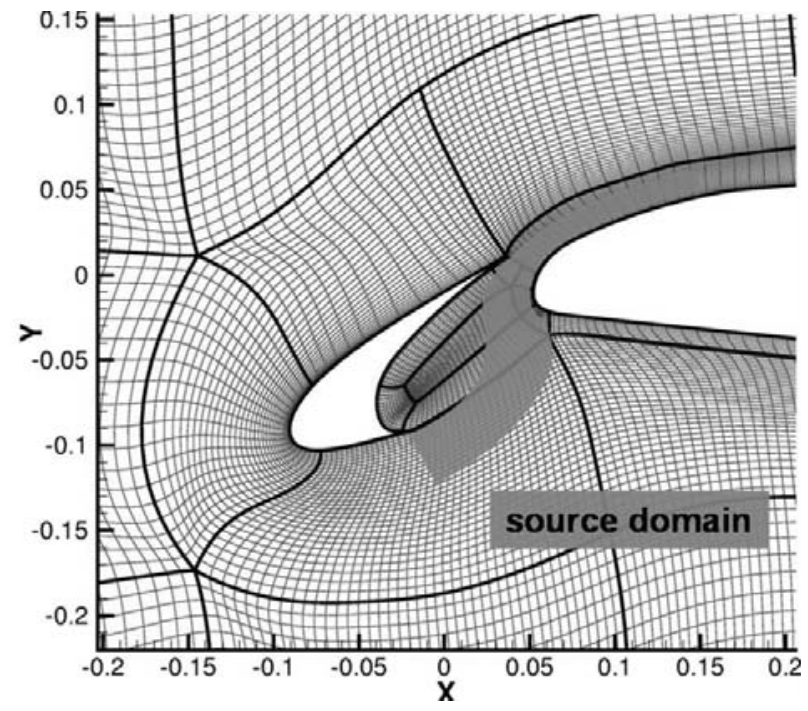

(b) Resolved source domain and curvilinear multi-block CAA mesh

Figure 1. Resolution of the slat shear-layer in the 2D test problem

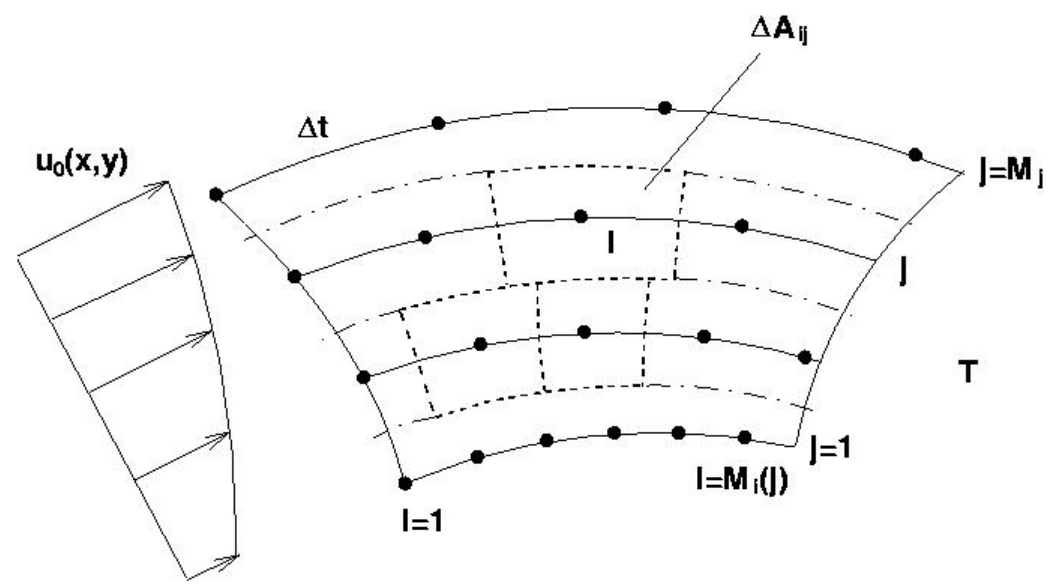

Figure 2. Sketch of streamlines and discrete points in non-uniform mean-flow 


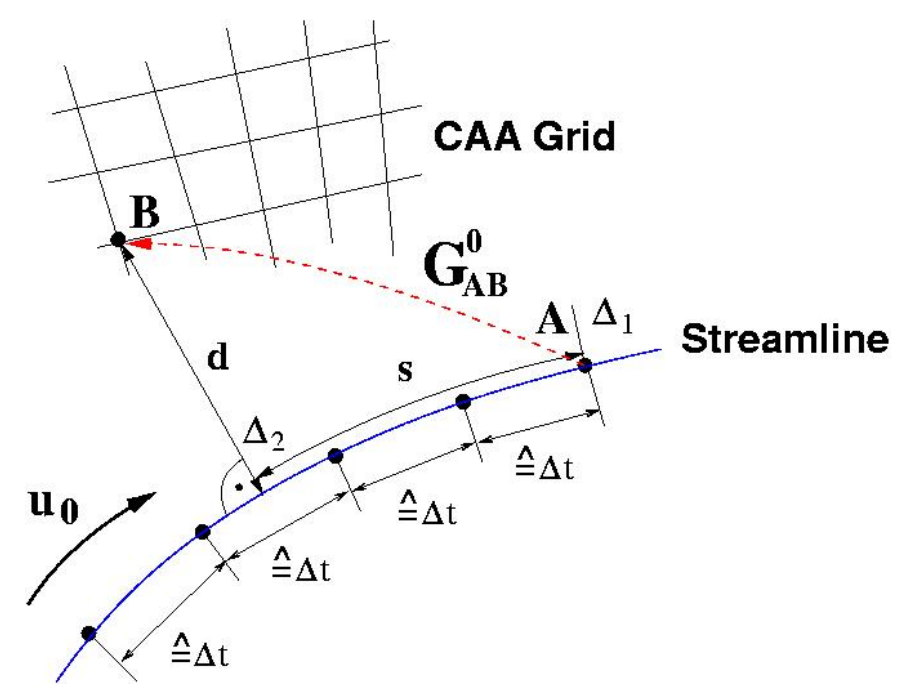

Figure 3. Interpolation onto CAA grid points.

For uniform flow with constant length-scale this is identical with the Gaussian kernel eq. (21). For curved streamlines a small distortion of the kernel shape occurs.

Note that each CAA grid point is linked to each streamline at most once, and the number of base-points is constant and independent of the number of discrete stochastic particle points along the streamline.

Usually, the small CAA time step would demand an unnecessary large number of discrete points along the streamline, if for each time increment the random numbers are shifted one full position downstream. In this work a larger spacing along the streamline was allowed by considering random particle positions between discrete points on the streamline. Then the interpolation onto the fixed points along the streamline is achieved through the first filter operation, whereby the filter is centered at the local particle position (i.e. it is moved with the random particle), but the filtered values are computed for fixed streamline points. The method can be called a random particle-mesh (RPM) method, where the random values carried by Lagrangian particles are interpolated onto the CAA grid using the filter kernel as locally defined shape functions.

\section{A. Scaling of the filter kernel parameters from RANS}

The filter kernel parameter $l_{\mathrm{s}}$ and $\hat{A}$ are scaled using a steady RANS computation. In general, based on the kinetic turbulent energy $\bar{k}$ and the mean dissipation rate $\epsilon$ an integral length scale can be deduced:

$$
l_{\mathrm{s}}=c_{l} \frac{\bar{k}^{3 / 2}}{\epsilon} .
$$

Following the discussion of Bailly \& Juvé ${ }^{13}$ the constant can be estimated to be $c_{l} \approx 0.54$ for a modified von Karman spectrum. Although a Gaussian spectrum is used in this work, the value for $c_{l}$ is also adopted here. Equating the turbulent viscosity $\nu_{T}$ of the $\bar{k}-\epsilon$ with that of a $\bar{k}$ - $\omega$ model, the relation $\epsilon=C_{\mu} \bar{k} \omega$ follows, where $C_{\mu}=0.09$. Then the length scale in terms of the $\bar{k}$ - $\omega$-model becomes

$$
l_{\mathrm{s}}=\frac{c_{l}}{C_{\mu}} \frac{\bar{k}^{1 / 2}}{\omega} \approx 6.00 \frac{\bar{k}^{1 / 2}}{\omega} .
$$

11 of 33 
The amplitude $\hat{A}$ is scaled by computing from eq. (17) the value of the kinetic turbulent energy and by relating this expression to the turbulent kinetic energy $\bar{k}$ of the RANS computation. Using eq. (17) and eq. (6) the velocity components read

$$
u^{\prime}=\iint \hat{A} \frac{\partial G^{0}}{\partial y} \mathcal{U} \mathrm{d} \boldsymbol{x}^{\prime}, \quad v^{\prime}=-\iint \hat{A} \frac{\partial G^{0}}{\partial x} \mathcal{U} \mathrm{d} \boldsymbol{x}^{\prime} .
$$

Using eq. (??), evaluated for $r=0$, the turbulent kinetic energy becomes

$$
\bar{k}=\frac{3}{4}\left(\overline{u^{2}}+\overline{v^{2}}\right)=\frac{3}{4} \iint \hat{A}^{2}\left[\left(\frac{\partial G^{0}}{\partial x}\right)^{2}+\left(\frac{\partial G^{0}}{\partial y}\right)^{2}\right] \mathrm{d} \boldsymbol{x}^{\prime} .
$$

Inserting the Gaussian kernel and neglecting the week spatial dependence of $\hat{A}$, the amplitude relates to the value of the turbulent kinetic energy via

$$
\hat{A}=\sqrt{\frac{4}{3 \pi}} \bar{k}^{1 / 2} \approx 0.651 \bar{k}^{1 / 2} .
$$

Note that the amplitude is independent of the local value of $l_{\mathrm{s}}$. It is completely determined by the kinetic turbulent energy.

\section{Acoustic simulation techniques}

For the acoustic simulations acoustic perturbation equations (APE-4) as introduced by Ewert \& Schröder $^{12}$ are used. They are a modification of the genuine linearized Euler equations (LEE). The system solved for the pressure and velocity perturbations $\left(p^{\prime}, \boldsymbol{u}^{\prime}\right)$ is

$$
\begin{aligned}
& \frac{\partial p^{\prime}}{\partial t}+c_{0}^{2} \boldsymbol{\nabla} \cdot\left(\rho_{0} \boldsymbol{u}^{\prime}+\boldsymbol{u}_{0} \frac{p^{\prime}}{c_{0}^{2}}\right)=c_{0}^{2} q_{c} \\
& \frac{\partial \boldsymbol{u}^{\prime}}{\partial t}+\nabla\left(\boldsymbol{u}_{0} \cdot \boldsymbol{u}^{\prime}\right)+\boldsymbol{\nabla}\left(\frac{p^{\prime}}{\rho_{0}}\right)=\boldsymbol{q}_{m} .
\end{aligned}
$$

Here $\rho_{0}, p_{0}$, and $\boldsymbol{u}_{0}$ denote the density, pressure and velocity of the time averaged flow, respectively. Furthermore, $c_{0}=\sqrt{\gamma p_{0} / \rho_{0}}$ is the local speed of sound. It is easy to show that the APE system eqs. $(40,41)$ exclude the vortical (hydrodynamical) modes by taking the curl of eq. (41). Considering the source term on the right-hand side, the vorticity equation of the APE system becomes

$$
\frac{\partial \boldsymbol{\omega}^{\prime}}{\partial t}=\boldsymbol{\nabla} \times \boldsymbol{q}_{m}
$$

Hence, the perturbation vorticity $\boldsymbol{\omega}^{\prime}=\boldsymbol{\nabla} \times \boldsymbol{u}^{\prime}$, on the left-hand side is completely controlled by the righthand side (RHS) source term. For the homogeneous system with all sources removed, the vorticity equation reduces to the statement that the vorticity remains constant (zero), i.e., unlike the LEE, the APE do not support the convective vorticity mode.

Rewriting the Navier-Stokes equations in non-linear disturbance formulation such that the left-hand side reassembles the APE system, the remaining terms lumped together on the right-hand side read ${ }^{12,14}$ (neglecting non-linear perturbation entropy terms)

$$
\begin{aligned}
& q_{c}=-\nabla \cdot\left(\rho^{\prime} \boldsymbol{u}^{\prime}\right)^{\prime}+\frac{\rho_{0}}{c_{p}} \frac{D_{0} s^{\prime}}{D t} \\
& \boldsymbol{q}_{m}=-(\boldsymbol{\omega} \times \boldsymbol{u})^{\prime}+T^{\prime} \nabla s_{0}-s^{\prime} \boldsymbol{\nabla} T_{0}-\left(\boldsymbol{\nabla} \frac{\left(u^{\prime}\right)^{2}}{2}\right)^{\prime}+\left(\frac{\boldsymbol{\nabla} \cdot \underline{\tau}}{\rho}\right)^{\prime},
\end{aligned}
$$


where $(\ldots)^{\prime}:=(\ldots)-\overline{(\ldots)}$ denotes the perturbation of terms. The major vortex source term is the fluctuating Lamb vector

$$
\boldsymbol{q}_{m}=-(\boldsymbol{\omega} \times \boldsymbol{u})^{\prime}=-\boldsymbol{\omega}_{0} \times \boldsymbol{u}^{\prime}-\boldsymbol{\omega}^{\prime} \times \boldsymbol{u}_{0}-\left(\boldsymbol{\omega}^{\prime} \times \boldsymbol{u}^{\prime}\right)^{\prime} .
$$

A similar vortex source term appears in the acoustic analogies of Powell, Howe, and Möhring. ${ }^{15,16,17}$ The source term is computed from the velocity fluctuations provided by the stochastic method. The equations are integrated with the DLR CAA code PIANO applying the 4 th order DRP scheme of Tam \& Webb in space $^{18}$ and a LDDRK method ${ }^{19}$ in time on block structured meshes.

\section{Computational results}

\section{A. Simulation features}

A modified two-element high-lift configuration has been used for the computations. Fig. 9(a) shows a cross section of the considered geometry. Four different CAA grids are used for the aeroacoustic computations. For the simulation of the Mach number scaling law a grid consisting of 25 blocks with about 200k mesh points is used. It solves the acoustic field in a $5 c \times 5 c$ box, where $c$ denotes the main-element chord length. Based on a dimensional chord length $c=0.4 \mathrm{~m}$, the grid is sufficient to resolve frequencies up to $12 \mathrm{kHz}$. The topology is of a mixed $H / C$-type. An $H$-topology is used in the outer region, which yields almost Cartesian cells in the far-field. A $C$-grid is used in the vicinity of the airfoil to resolve the airfoil with the slat. The mesh resolution is enhanced in the slat-cove to resolve the estimated length scale of the source term properly. A refined version of the grid with about 550k mesh points is also used.

Two different grids are used for the slat gap trend predictions. A coarse grid which consists about 100k mesh points resolves a $6 \times 7 c$ box. It consists of 16 blocks and is capable to resolve frequencies up to $5 \mathrm{kHz}$. The topology is similar to the $250 \mathrm{k}$ grid and is used unchanged for the three different considered slat settings. Furthermore a refined version of the grid is applied with doubled grid density in each direction. Hence, it consists of about $400 \mathrm{k}$ grid points and has a resolution limit around $10 \mathrm{kHz}$.

The RANS mean-flow fields are computed with the DLR flow-solver TAU on an unstructured mesh. The free stream Mach number considered in this paper are $M=0.088,0.10,0.12$, and 0.16. A Menter SST turbulence model $^{20}$ with Kato-Launder modification ${ }^{21}$ is used.

\section{B. Reference slat configurations}

First, results for the reference two-element airfoil are presented for a mean-flow velocity of $M=0.10$. Fig. 4 presents narrow band spectra for two grids with different resolution for an observer position above the slat. Furthermore, the computed narrow band spectra are juxtaposed to an acoustic far-field measurement that has been conducted by EADS Corporate Research Centre in DLR's Acoustic Windtunnel Braunschweig (AWB) as part of the German national project FREQUENZ. A qualitative good agreement in the spectral distributions is obtained. As expected, the coarse grid solution falls off the measured spectral trend, reaching a highest frequency of approximately $12 \mathrm{kHz}$. Since the coarse grid corresponds to the previously discussed $250 k$ grid, this finding confirms the resolution estimate initially made for it. The finer grid solution follows the measured narrow band solution almost over the whole range of meaningful frequencies up to $20 \mathrm{kHz}$.

Fig. 5 compares an APE/RPM based 1/3-octave spectrum with the empirical slat-noise model of Dobrzynski. ${ }^{1,22}$ The empirical slat-noise model is based on a generic spectrum that shows a typical peak frequency and falls off for higher frequencies according to a $f^{-1.8}$ law. The simulation reproduces these two distinct features, i.e., the peak frequency in a similar frequency range and the rate of decay of the spectrum for higher frequencies.

The influence of the length-scale parameter $c_{l}$, eq. (37), on the acoustic far-field and the turbulent nearfield spectra is studied in Fig. 6. Acoustic simulations have been carried out for two values of $c_{l}$, i.e., a theoretically motivated value of $c_{l}=6.0$, Section $\mathrm{A}$, and a clearly reduced value of $c_{l}=2.0$. The dashed lines in Fig. 6 correspond to the longitudinal velocity correlations induced by the model in a representative 
point in the shear layer. According to the underlying filter kernel, their spectral distributions follow the shape of a Gaussian. Obviously, due to the three times smaller structures in case $c_{l}=2.0$, the related spectrum is stretched to (three times) higher frequencies. Accordingly, the characteristic spectral fall off occurs at higher frequencies. An astonishing effect is found for the acoustic far-field spectra, plotted with solid lines. They exhibit a common narrow band fall off in the range between $f^{-2}$ and $f^{-3}$, with just a small absolute difference of about $3 \mathrm{~dB}$ over a large range of frequencies. Apparently, the shape of the acoustic spectrum is not directly influenced by the shape of the turbulence spectrum and supports a completely different decay law. Therefore, the length-scale parameter has almost no effect on the acoustic spectra.

An explanation for this unexpected effect could be based on the 'incompressible upwash velocity' concept introduced by Howe in. ${ }^{23}$ Howe argues that the upwash velocity in the vicinity of a flat plate trailing edge, which is determined by the Biot-Savart induction formula applied to the boundary layer vorticity outside the viscous sublayer, is the appropriate metric that characterizes structure born sound generation in low Mach number flows. Since a kinematic boundary condition holds at the surface, the surface will 'respond' with a wall normal velocity that cancels the upwash velocity, eventually giving rise to sound radiation. Fig. 7 sketches the upwash velocity field $v_{n}$ induced by a vortex of characteristic size $\Delta$ that passes a plate in distance $h$ at convection speed $u_{c}$. It is evident that the characteristic frequency induced by moving the upwash pattern with $u_{c}$ over the trailing edge is determined by the miss distance $h$, i.e.,

$$
f \propto \frac{u_{c}}{h},
$$

and not by the characteristic eddy size $\Delta$.

\section{Mach number scaling law}

Results of the Mach number scaling study are plotted in Fig. 8. Three different Mach numbers $(0.088,0.12,0.16)$ are considered. The Figs. 8(a)-(c) show instantaneous pressure plots for fixed contour levels. The acoustic fields roughly correspond to the one expected for a dipole source placed at the slat trailing edge with its axis normal to the slat chord. A clear increase in the amplitude of the radiated pressure waves is visible for an increasing flow velocity. From experiments it is deduced that the sound intensities scale according to a $M^{4.5}$ law for slat-noise, i.e., a slightly weaker growth compared to airfoil trailing edge noise, which can be shown analytically to scale with $M^{5}$. Usually the exponent can be attributed to several effects. First, the change of the convection velocity of vortical structures causes an increase in the radiated sound pressure. Second, the turbulent velocity fluctuations grow with increasing flow velocity. For analytical considerations it is usually assumed that the turbulent velocity fluctuation scale linearly with the freestream velocity. In the stochastic model the convection property is accounted for by convecting the random particles in the steady RANS mean-flow. The increase of turbulent fluctuations is taken into account in the model by the change of the turbulence kinetic energy levels, which are provided by the steady RANS solution. The Fig. 8(d) depicts the scaling of the root-mean square (RMS) fluctuating pressure for an observer position 1.5c below the slat trailing edge. A linear regression fit to the three data points yields a Mach number scaling law exponent of 1.54 for the RMS pressure. Just considering the first two Mach numbers the exponent changes to 1.65. Since the sound intensities scale with twice that value, one finds a scaling law with exponents between 3.08 and 3.3 for the considered 2D case. In Ref. ${ }^{24}$ it was discussed that a $2 \mathrm{D}$ acoustic solution has to be corrected to take into account 3D sound radiation, which increases the scaling exponent by one. The correction formula was deduced to read

$$
\widetilde{p}(0, R, \theta, \omega) \simeq \hat{p}(R, \theta, \omega) \frac{1+i}{2} \sqrt{\frac{k L^{2}}{\pi R}} .
$$

Here $\tilde{p}$ denotes the $3 \mathrm{D}$ corrected (complex) spectral pressure and $\hat{p}$ is related to the $2 \mathrm{D}$ spectral pressure. $L$ denotes a length scale of the problem, see Ref. ${ }^{12}$ Based on Taylor's hypothesis of convecting vorticity, the non-dimensionalizing of Eq. (46) yields with $k=\omega / c, \omega \propto u / \lambda_{v}$, where $\lambda_{v}$ is the length scale of a vortical disturbance, the scaling

$$
\widetilde{p} \propto M^{1 / 2} \hat{p}
$$


i.e., the sound intensities in 3D scale with the power of the Mach number increased by one, compared to the $2 \mathrm{D}$ case. Accordingly, the corrected 3D scaling would become 4.08 and 4.3 , which is pretty close to the expected exponent.

\section{Slat gap variations}

As a next problem the acoustic effects of a slat gap variation is studied. Three different slat settings are considered, which are depicted in Fig. 9(b). The slat gaps are varied by moving the slat in vertical direction (unchanged overlap). Beside a reference gap configuration, which is conformal with the previously discussed configuration and also is shown in Fig. 9(a), an increased gap (145\% reference gap width) and a reduced gap ( $85 \%$ reference gap width) are considered. The source patches that have been used to resolve the turbulent slat cove source are presented in Fig. 10. On the source patches the turbulence kinetic energy is plotted. The resolved source region excludes the recirculation bubble. Hence, the computations might help to identify whether the unsteady recirculation bubble is the major slat-noise source or if the convecting vortical disturbances that pass the slat gap and trailing edge are the major cause for slat-noise. Near-field details of the turbulent flow field, which is provided by RANS solutions of the DLR TAU code, are depicted in the Figs. 11, 12, and 13 for the three slat settings. The figures respectively show the turbulence kinetic energy, the mean-flow velocity, and the integral length scale based on eq. (37). Hence, the main quantities that affect the velocity fluctuations in the stochastic model and thus the sound generation and radiation. The abbreviation TE indicates the lower corner of the slat trailing edge. Fig. (11) plots logarithmic energy levels in $\mathrm{dB}$. For the reduced and reference gap configurations similar turbulence kinetic energy levels are visible in the slat gap. Also the mean-flow velocity has equal magnitudes in both cases with approximately $65 \mathrm{~m} / \mathrm{s}$ along the contour-line through the trailing edge for the reference gap configuration and about $60 \mathrm{~m} / \mathrm{s}$ for the reduced gap case. For the increased gap width slightly higher values for the turbulence energy levels are visible close to the trailing edge compared to the reference case. Also in contrast to the reference solution the energy levels decay much more rapidly with increasing distance to the slat lower side. For the larger gap width case clearly reduced turbulence levels are visible at the main element surface. The gap velocity is increased for the larger gap width compared to the reference solution.

An important feature that has to be accomplished by the stochastic model is the accurate resolution of the turbulence kinetic energy topology. To evidence this capability of the model, the Figs. 14 and 15 juxtapose the RANS target solutions for the turbulence energy to the according results from the stochastic models. For this purpose, 20000 time levels of the stochastically generated velocities are sampled and averaged. One free parameter of the model is the number of random particles that are used to set up the fluctuating white-noise field. This number is determined by the number of mean-flow streamlines to resolve the source patch and the number of random points along the streamlines (the time increment along the streamline, see Fig. 3). The influence of the number of streamlines to resolve the source region is highlighted in Fig. 14 for the reference slat gap configuration. 30, 50, and 100 streamlines have been considered for this test. A fairly good realization of the turbulence energy for the energy topology as well as the absolute magnitudes is found. The streamtrace variations evidence only a weak dependence on the free parameter of the stochastic model. Fig. 15 gives similar results for the other two slat gap settings, using 30 streamlines. Again, the model yields a fairly good agreement in the topology and absolute values compared to the RANS target solutions.

Instantaneous pressure plots for the three slat gap settings are presented in Fig. 16.

Narrow band spectra for four different polar angles are presented in Fig. 17. The observer positions are located on a circle of radius 1.5 chord lengths, which is centered at the slat trailing edge. A polar angle of $\theta=90^{\circ}$ corresponds to an observer position above the trailing edge. The other three polar angles resolve the arc that is interesting for sound radiation towards the ground. The spectra are being based on the coarse grid solution, which are sufficient to resolve frequencies up to 6 to $8 \mathrm{kHz}$. This grid feature is indicated in the spectra by their rapid fall-off in this frequency range. Fig. 18 compares the coarse grid spectra with those obtained from a fine grid solution with doubled grid density in each direction. It can be seen that the fine grid solutions clearly double the resolved frequency range. However, in the resolved frequencies 
both coarse and fine grid spectra agree closely in their distribution and their amplitudes, independent of the considered gap configuration. Fig. 19(a) gives results for the narrow band spectra using different numbers of streamlines to resolve the turbulent source patch. According to the findings for the turbulent kinetic energy reconstruction, only a weak dependence on the model parameter is found. Fig. 19(b) presents the pressure history for a receiver point in the CAA domain over 50000 CAA time steps. The plot evidences a stable result without any drift in the mean values or amplitudes over time.

No clear trend is apparent by comparing the sound pressure levels of the different slat gap settings, Fig. 17. From the experiments of Pott-Pollenske et al. ${ }^{25}$ it is known that a reduced gap width can have a significant effect on the acoustic far-field. As was shown in Ref., ${ }^{25}$ a reduction of the slat gap width of about $15 \%$ caused a decrease about $5 \mathrm{~dB}$ of the sound pressure levels over all frequencies. However, based on the close correspondence of the values in the RANS solutions, in particular for the small and large slatgap settings, which was evidenced by the Figs. 11, 12, it appears reasonable that these two configurations exhibit almost similar acoustic spectra. To analyze the findings further, Fig. 20 presents RANS solutions for the current flapless two-element airfoil configuration and the three-element configuration used by PottPollenske et al. Fig. 20(a) identifies the cuts along which RANS data have been extracted. UTE,P denotes a point at the upper slat trailing edge on the pressure (cove) side. In the studies of Pott-Pollenske et al. it was found that the velocities close to this point were most appropriate to scale the experimental acoustic data. Fig. 20(b) depicts the velocity distribution for the three-element configuration for a intersection line normal to the slat surface, starting from UTE,P. In particular the velocities in the range around $0.25 \mathrm{~mm}$ were considered to characterize the sound generation process. Clearly reduced velocity levels are present for the slat configuration with reduced gap in Fig. 20(b) and explains the reduced sound levels for this case. Fig. 20(c) depicts a similar velocity distribution for the current flapless two-element airfoil case. In particular in the range around $0.25 \mathrm{~mm}$ the reduced gap width velocity distribution reaches even slightly higher velocities compared to the reference solution. Furthermore, the turbulence kinetic energy distribution along the intersection line, as depicted in Fig. 20(d), even collapses for two slat gap settings. Hence, the acoustic solution that shows only a small difference between these two configurations is explainable based on the underlying RANS solutions. However, the question remains how the large differences in the slat gap flows between the two-element and the three-element configuration occurs. Chances are that the local flow situation is changed significantly going from a full three-element configuration to a two-element airfoil by removing the flap. This points to the importance of incorporating the flap for aeroacoustic predictions of slat noise.

However, the larger slat gap setting produces in the RANS solution higher local flow velocities close to UTE,P as well as higher levels of turbulent kinetic energy. Hence, it is reasonable that the acoustic spectra also feature higher sound pressure levels compared to the reference solution. However, note that the turbulence kinetic energy topology for the large gap setting differs significantly from that of the reference solution. In particular, Fig. 20(d) shows a much more rapid decay with the average energy along the intersection line having lower values compared to the reference case. Furthermore, in the small and reference gap cases the higher amount of fluctuating turbulence close to the main element surface could give rise to sound radiation due to the interaction with the main element. Due to the more reduced energy levels at the main element for the large slat gap setting, the interaction noise sources at the main element would be smaller.

Over all polar angles normalized directivities are depicted in the Figs. 21 and 22. For a frequency of $1 \mathrm{kHz}$ coarse and fine grid directivities agree quantitatively well. The $1 \mathrm{kHz}$ directivity exhibits the same characteristics as discussed by Ewert \& Emunds. ${ }^{3}$ The root-mean-square directivity is shown in Fig. 23 for the coarse grid solution. A dipole like directivity pattern is visible with its axis tilted in accordance with the slat deflection. The large gap directivities shows slightly higher values, followed by the reference and the reduced gap directivities. 


\section{Conclusions}

Slat Noise simulations have been carried out for a high-lift airfoil. The high-lift airfoil geometry is a two-element slat-airfoil configuration without deployed flap in order to isolate slat noise and avoid additional sound sources at the flap. The 3D Mach number scaling law exponent of the broadband slat noise component is evaluated from three different freestream velocities $(M=0.088,0.118,0.165)$ to be in the range 4.08 and 4.3 , which corresponds fairly good with experimental findings that show an exponent around 4.5. The capability of the stochastic method to reproduce a target turbulence kinetic energy topology is shown. The effect of streamline density variations in the stochastic model on the quality of the reproduced turbulent kinetic energy distribution and its effect on the predicted far-field spectrum is evaluated. Only a small dependence on the model parameter is found. The effect of slat gap variation on the broadband acoustic far-field is studied for three different slat configurations, which encompass beside the reference slat position one configuration with reduced, and one with increased slat gap. Previous experimentally findings at a full three-element high-lift airfoil, indicating a reduction of typically $5 \mathrm{~dB}$ for a slat gap reduction of about $15 \%$ could not be found for the flapless two-element configuration used. The acoustic findings are backed by the RANS slat flow simulations for the two-element geometry. They exhibit neither for the characteristic velocity nor for the turbulence kinetic energy a significant difference between the reference and the reduced gap configuration. It is likely that the local flow situation is changed significantly going from a full three-element configuration to a two-element airfoil by removing the flap. This points to the importance of incorporating the flap for aeroacoustic predictions of slat noise.

\section{Acknowledgment}

The author would like to thank Manuel Münsch and Michael Pott-Pollenske for their valuable contribution to this work. The RANS flow data where kindly provided by R. Emunds of Airbus Deutschland.

\section{References}

${ }^{1}$ Dobrzynski, W. and Pott-Pollenske, M., "Slat Noise Studies for Farfield Noise Prediction," 2001, AIAA Pap. 2001-2158.

${ }^{2}$ Singer, B., Lockard, D., and Brentner, K., "Computational Aeroacoustics Analysis of Slat Trailing-Edge Flow," AIAA Journal, Vol. 38(9), 2000.

${ }^{3}$ Ewert, R. and Emunds, R., "CAA Slat Noise Studies Applying Stochastic Sound Sources Based On Solenoidal Digital Filters," 2005, AIAA Pap. 2005-2862.

${ }^{4}$ Smirnov, A., Shi, S., and Celik, I., "Random Flow Generation Technique for Large Eddy Simulations and Particle Dynamics Modeling," Journal of Fluids Engineering, Vol. 123, 2001, pp. 359-371.

${ }^{5}$ Khavaran, A. and Bridges, J., "Modelling of fine-scale turbulence mixing noise," Journal of Sound and Vibration, Vol. 279, 2005, pp. 1131-1154.

${ }^{6}$ Tam, C. and Auriault, L., "Jet Mixing Noise from Fine-Scale Turbulence," AIAA Journal, Vol. 37(2), 1999, pp. 145-153.

${ }^{7}$ Davies, P., Fisher, M., and Barratt, M., "The Characteristics of the Turbulence in the Mixing Region of a Round Jet," J. Fluid Mech., Vol. 15, 1963, pp. 337 - 367.

${ }^{8}$ Morris, P. and Farassat, F., "Acoustic Analogy and Alternative Theories for Jet Noise Prediction," AIAA Journal, Vol. 40(4), 2002, pp. 671-680.

${ }^{9}$ Agarwal, A. and Morris, P., "Prediction Method for Broadband Noise from Unsteady Flow in a Slat Cove," AIAA Journal, Vol. 44(2), 2006, pp. 301-310.

${ }^{10}$ Careta, A., Sagués, F., and Sancho, J., "Stochastic generation of homogeneous isotropic turbulence with well-defined spectra," Physical Review E, Vol. 48(3), 1993, pp. 2279-2287.

${ }^{11}$ Batchelor, G., The Theory of Homogeneous Turbulence, Cambridge University Press, 1960.

${ }^{12}$ Ewert, R. and Schröder, W., "Acoustic Perturbation Equations Based on Flow Decomposition via Source Filtering," J. Comput. Phys., Vol. 188, 2003, pp. 365-398.

${ }^{13}$ Bailly, C. and Juve, D., "A Stochastic Approach To Compute Subsonic Noise Using Linearized Euler's Equations," 1999, AIAA Pap. 99-1872.

${ }^{14}$ Ewert, R. and Schröder, W., "On the simulation of trailing edge noise with a hybrid LES/APE method," Journal of Sound and Vibration, Vol. 270, 2004, pp. 509-524.

${ }^{15}$ Powell, A., "Theory of vortex sound," Journal of the acoustical society of america, Vol. 36/1, 1964, pp. 177-195. 
${ }^{16}$ Howe, M. S., "Contributions to the Theory of Aerodynamic Sound, with Application to Excess Jet Noise and the Theory of the Flute," J. Fluid Mech., Vol. 71, No. 4, 1975, pp. 625-673.

${ }^{17}$ Möhring, W., "Modelling Low Mach Number Noise," Mechanics of Sound Generation in Flows, edited by E.-A. Müller, Springer, 1979.

${ }^{18}$ Tam, C. and Webb, J., "Dispersion-Relation-Preserving Finite Difference Schemes for Computational Acoustics," J. Comp. Phys., Vol. 107, 1993, pp. 262-281.

${ }^{19} \mathrm{Hu}$, F. Q., Hussaini, M. Y., and Manthey, J. L., "Low-dissipation and Low-Dispersion Runge-Kutta Schemes for Computational Acoustics," J. Comp. Phys., Vol. 124, 1996, pp. 177-191.

${ }^{20}$ Menter, F., "Zonal Two Equation k- $\omega$ Turbulence Models for Aerodynamic Flows," 1993, AIAA Pap. 93-2906.

${ }^{21}$ Kato, M. and Launder, B., "The modelling of turbulent flow around stationary and vibrating square cylinders," 9th symposium on turbulent shear flows, Kyoto, Japan, August 16th-18th, 1993, 1993.

${ }^{22}$ Pott-Pollenske, M., Dobrzynski, W., Buchholz, H., Gehlhar, B., and Walle, F., "Validation of a Semiempirical Airframe Noise Prediction Method through Dedicated A319 Flyover Noise Measurements," 2002, AIAA Pap. 2002-2470.

${ }^{23}$ Howe, M., "Trailing Edge Noise At Low Mach Numbers," Journal of Sound and Vibration, Vol. 225(2), 1999, pp. 211-238.

${ }^{24}$ Ewert, R., Zhang, Q., Schröder, W., and Delfs, J., "Computation of Trailing Edge Noise of a 3D Lifting Airfoil in Turbulent Subsonic Flow," 2003, AIAA Pap. 2003-3114.

${ }^{25}$ Pott-Pollenske, M., Alvarez-Gonzales, J., and Dobrzynski, W., "Effect of Slat Gap on Farfield Radiated Noise and Correlation with Local Flow Characteristics," 2003, AIAA Pap. 2003-3228. 


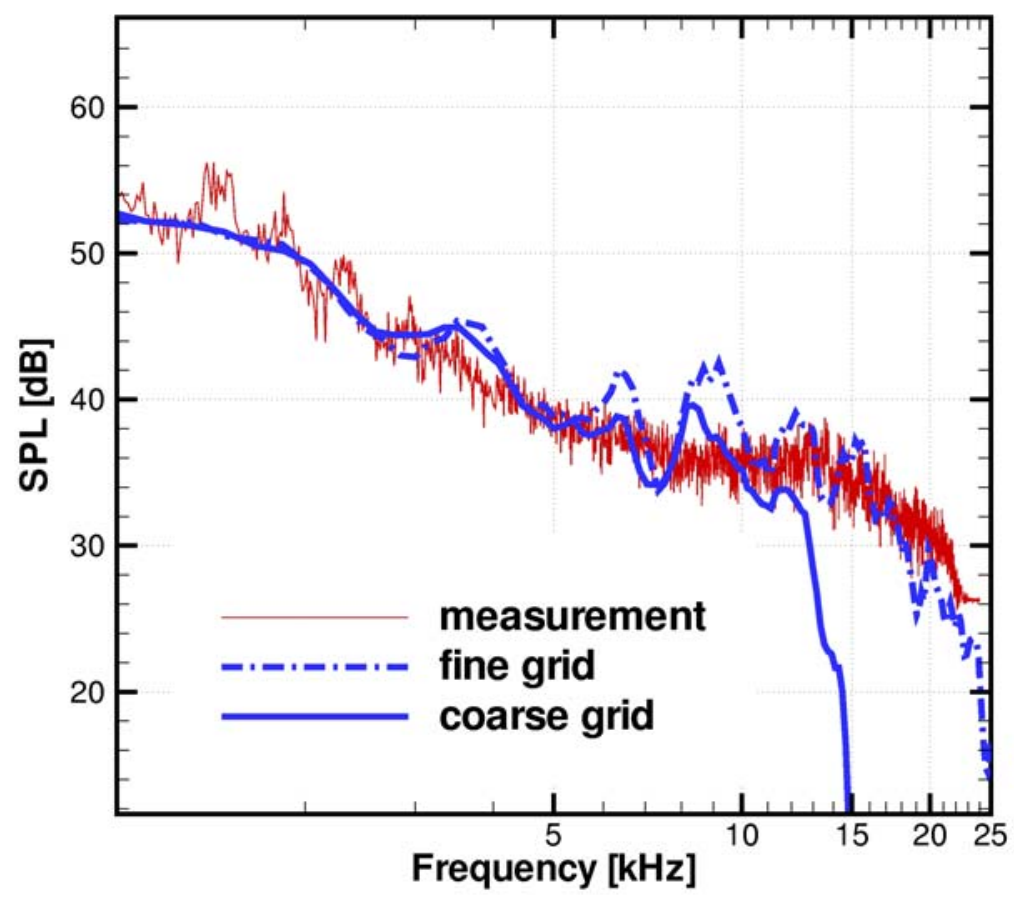

Figure 4. Comparison of APE/RPM based narrow band spectra on fine/coarse CAA meshes with acoustic windtunnel measurement.

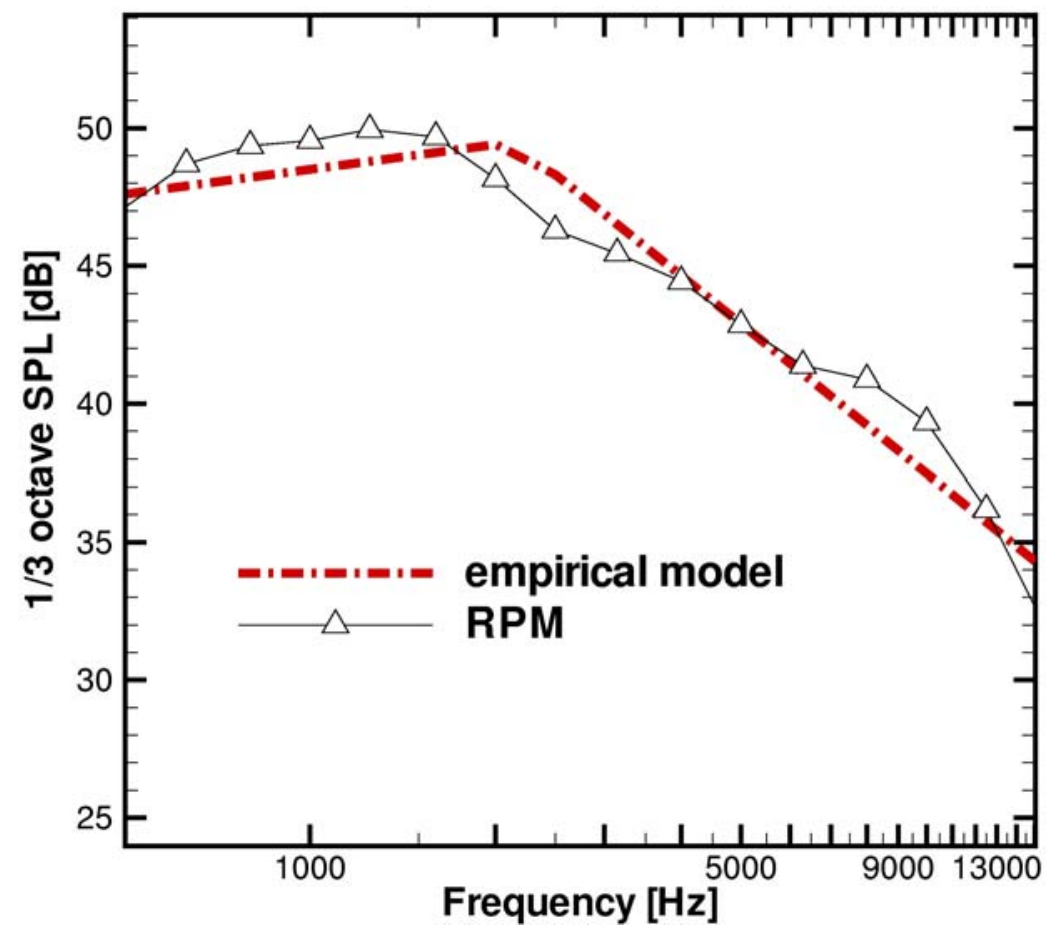

Figure 5. Comparison of APE/RPM based 1/3-octave spectrum with the empirical model spectrum of Dobrzynski. $^{1,22}$

19 of 33 


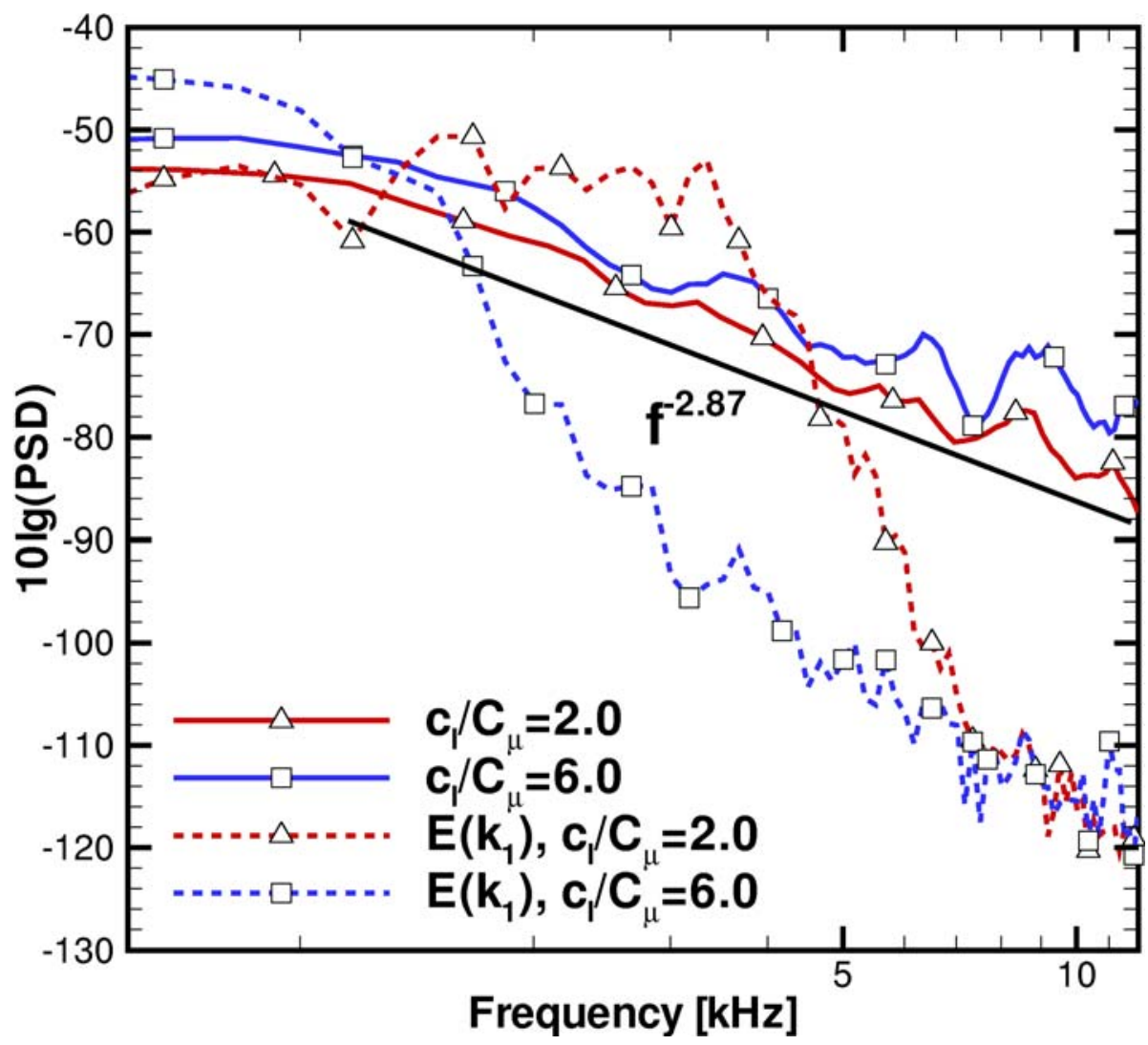

Figure 6. Influence of length-scale parameter $c_{l}$, eq. (37), on the acoustic far-field and the turbulent near-field spectra.

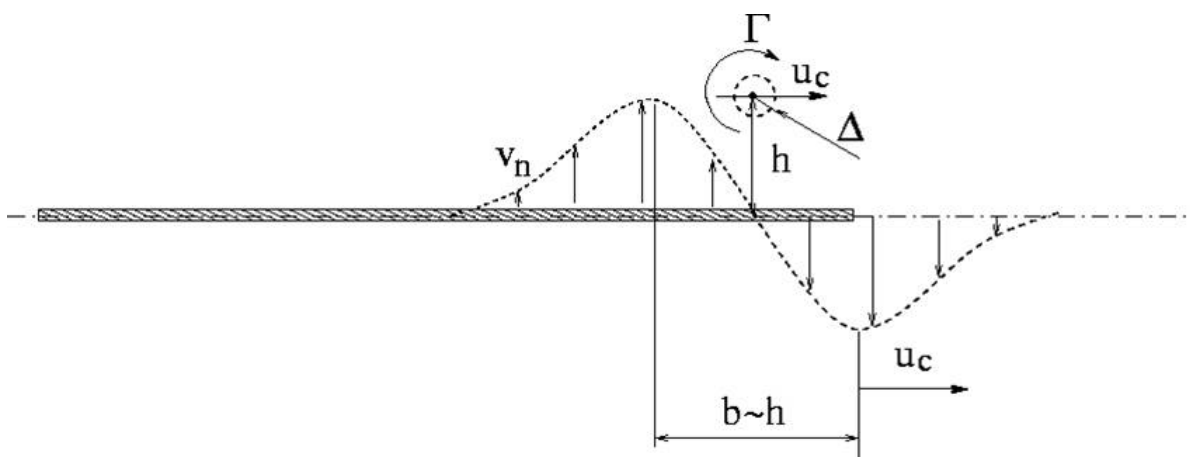

Figure 7. Incompressible upwash velocity concept; ${ }^{23}$ the characteristic frequency is determined by the miss distance $h$ as characteristic length scale, i.e., $f \propto u_{c} / h$ - not the eddy size $\Delta$. 


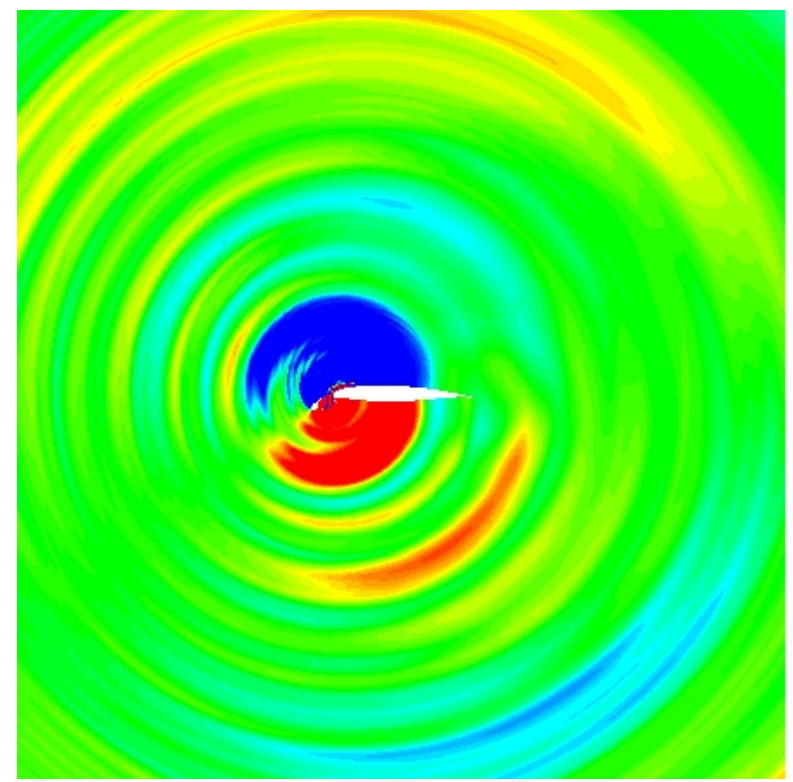

(a) $30 \mathrm{~m} / \mathrm{s}$

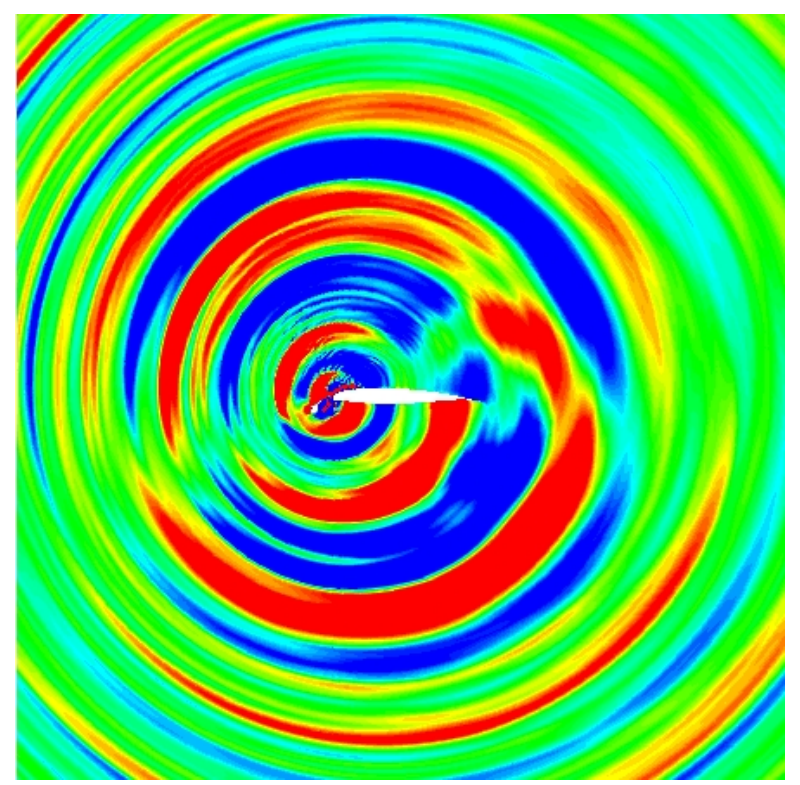

(c) $56 \mathrm{~m} / \mathrm{s}$

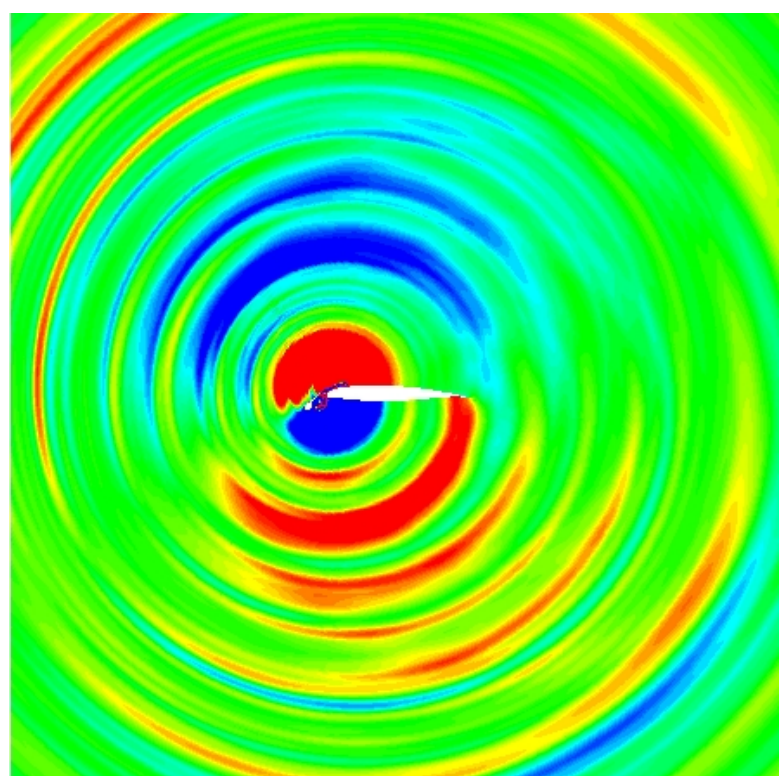

(b) $40 \mathrm{~m} / \mathrm{s}$

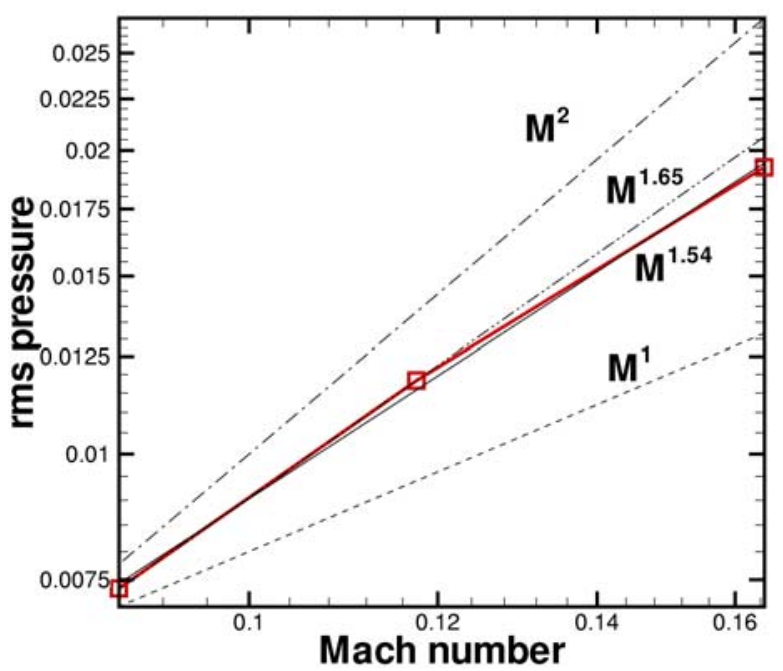

(d) Mach number scaling

Figure 8. Instantaneous pressure contours for different freestream velocities (with fixed contour scaling) and Mach number scaling in an observer point at $\theta=270^{\circ}, 1.5$ chord length below the slat trailing edge. 


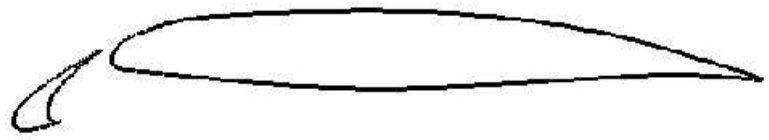

(a) Two-element high-lift airfoil

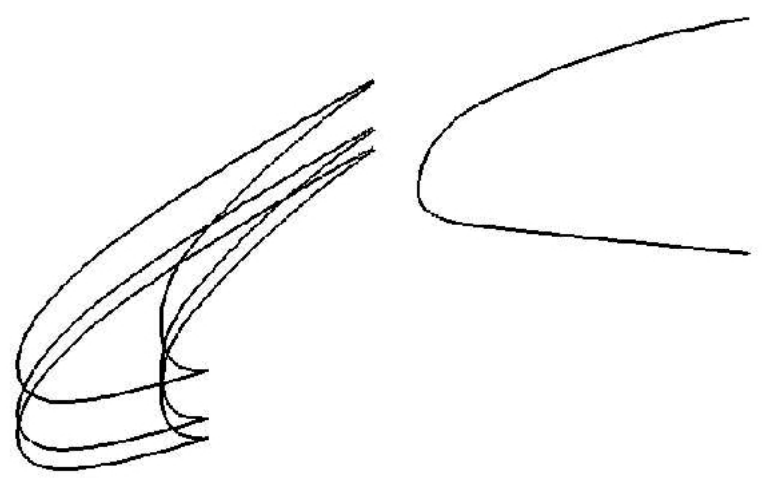

(b) Slat gap variations

Figure 9. Two-element slat configuration (a) and three slat gap settings (b) $(145 \%, 100 \%$, and $87 \%$ slat gap).

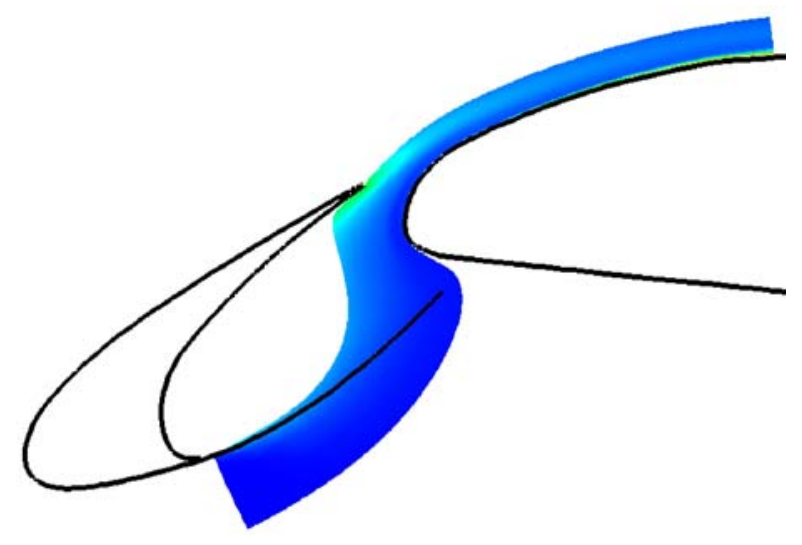

(a) Smaller gap width

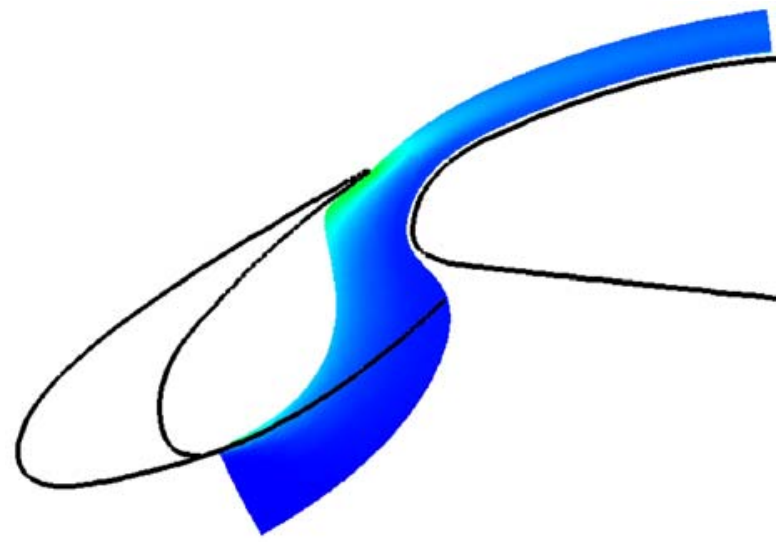

(b) Reference gap width

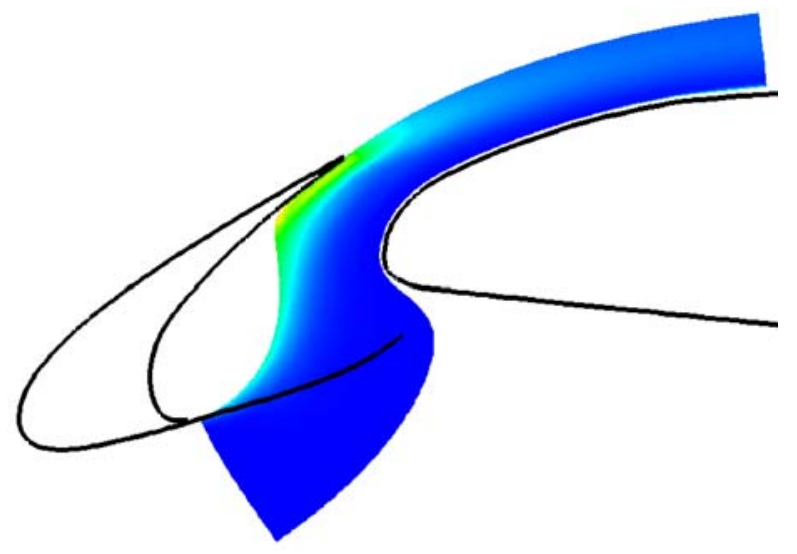

(c) Larger gap width

Figure 10. Stochastic source patches of the three slat configurations

$$
22 \text { of } 33
$$

American Institute of Aeronautics and Astronautics 


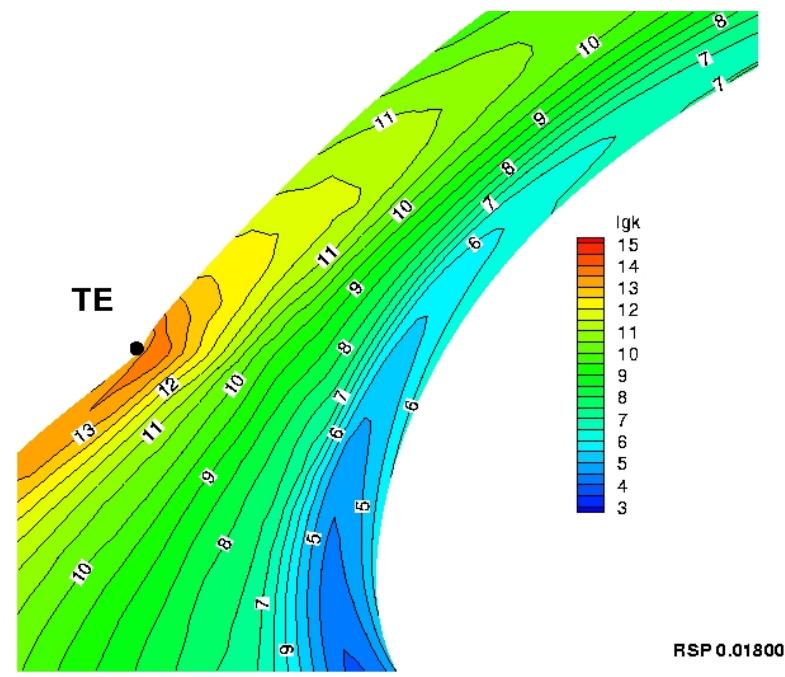

(a) Smaller gap width

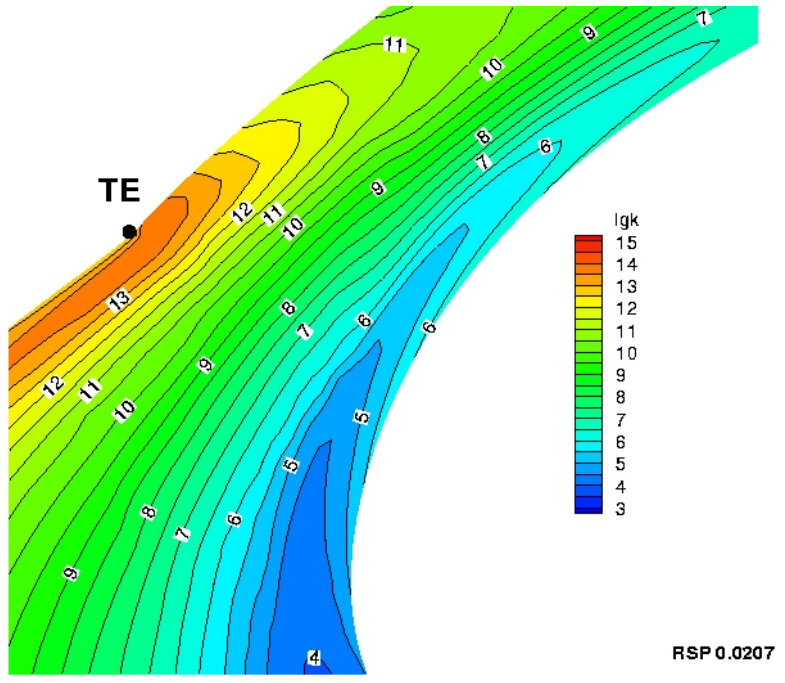

(b) Reference gap width

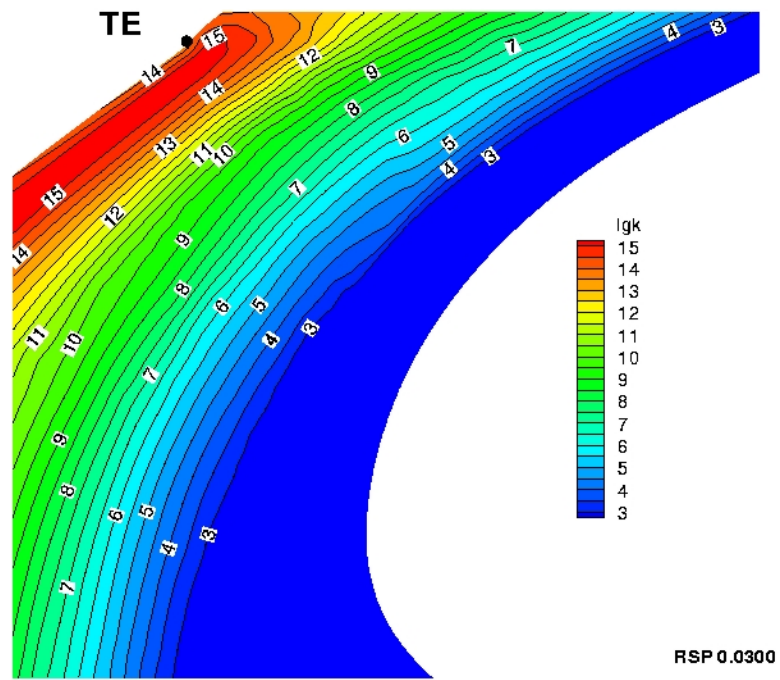

(c) Larger gap width

Figure 11. RANS solution of the turbulence kinetic energy in the slat gap $(\mathrm{TE}=$ trailing edge) 


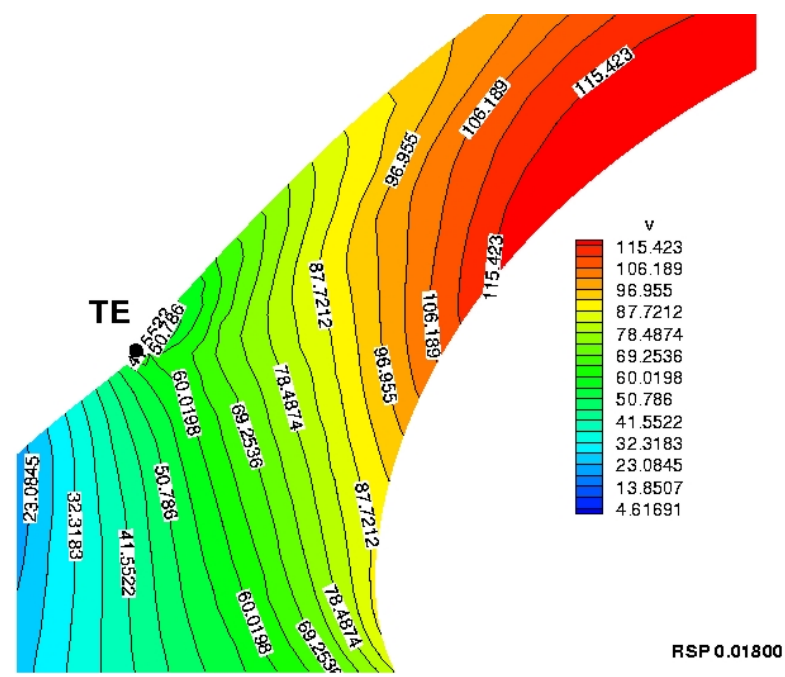

(a) Smaller gap width

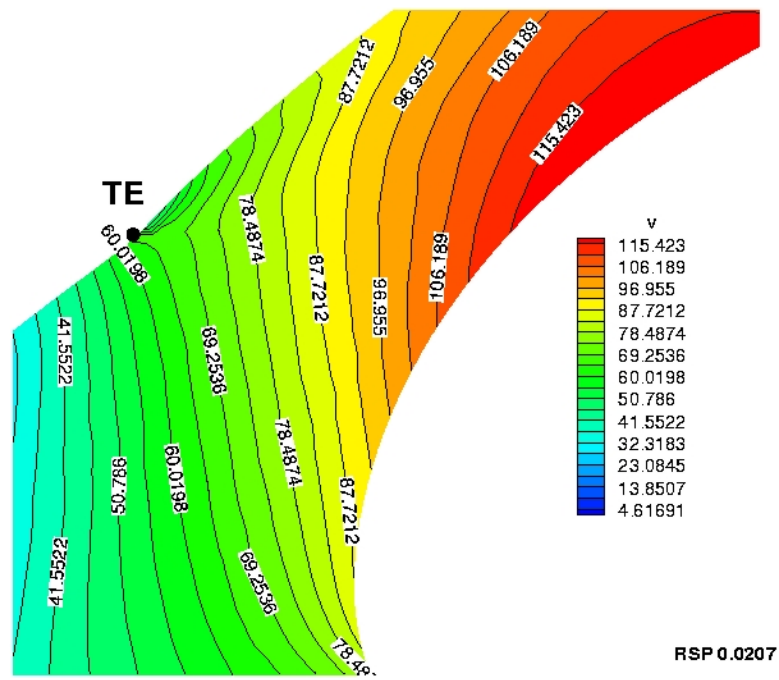

(b) Reference gap width

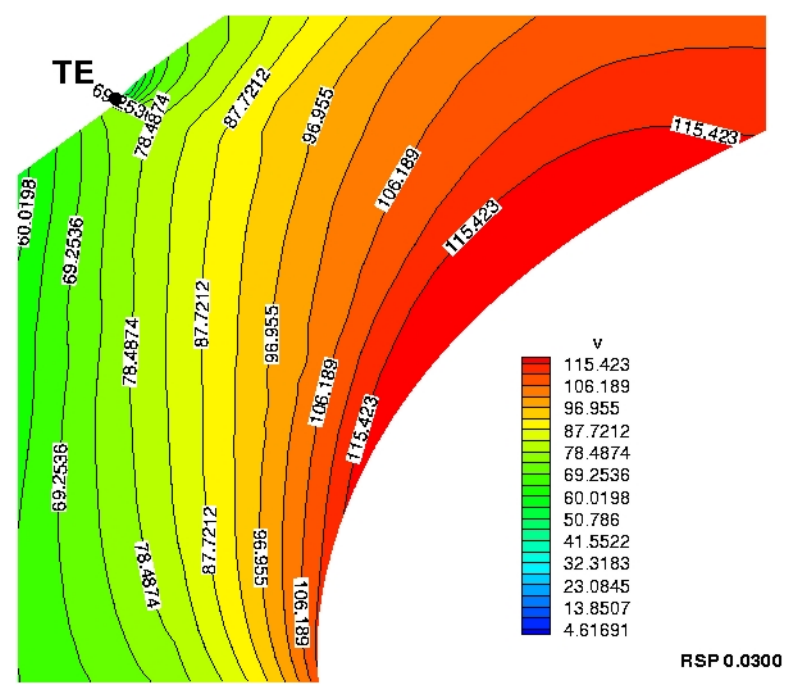

(c) Larger gap width

Figure 12. RANS solution of the mean-flow velocity in the slat gap ( $\mathrm{TE}=$ trailing edge) 


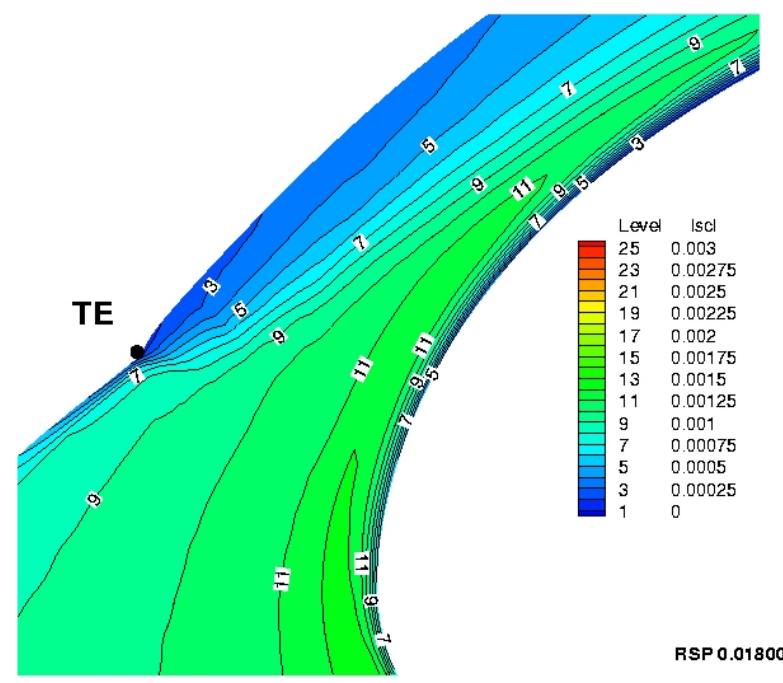

(a) Smaller gap width

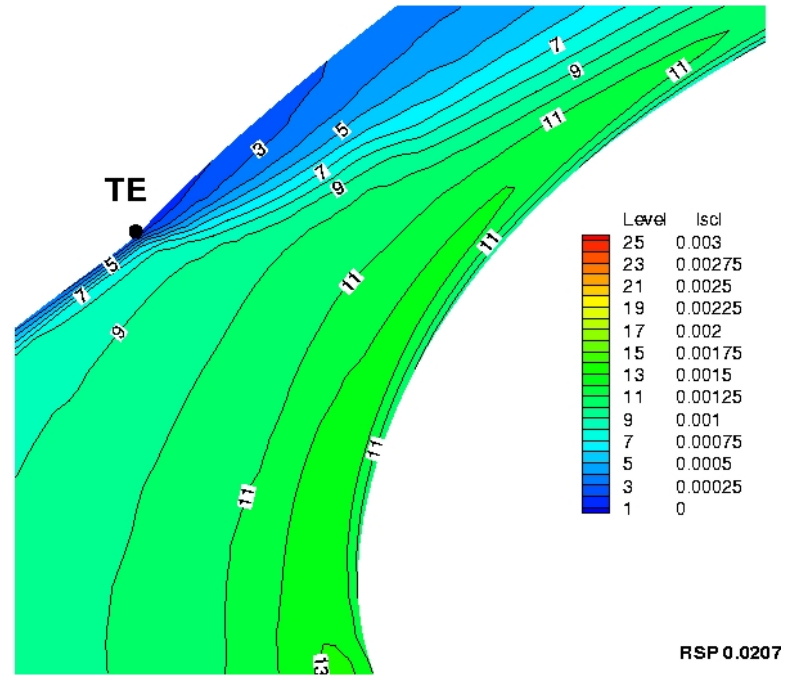

(b) Reference gap width

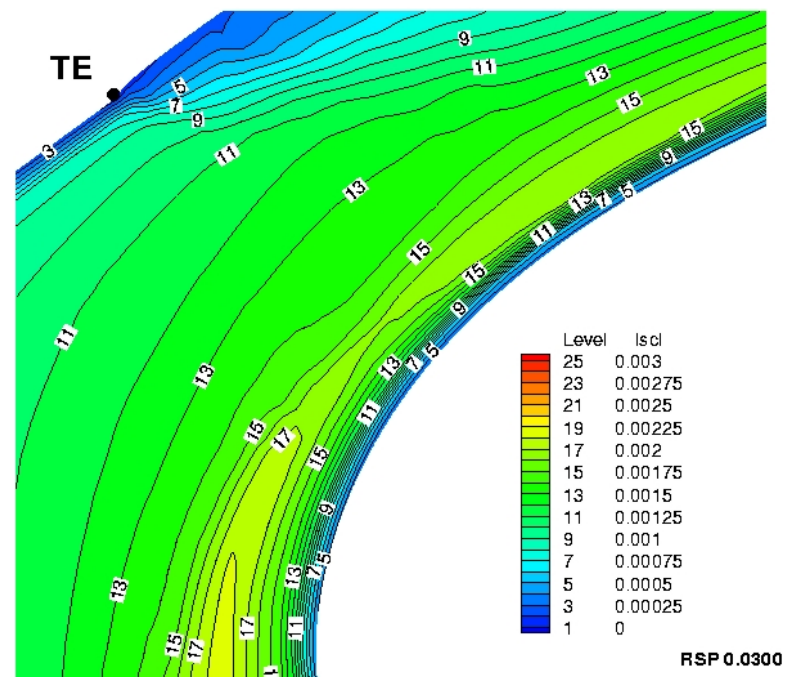

(c) Larger gap width

Figure 13. RANS solution of the integral length scale, eq. (37), in the slat gap ( $\mathrm{TE}=$ trailing edge) 


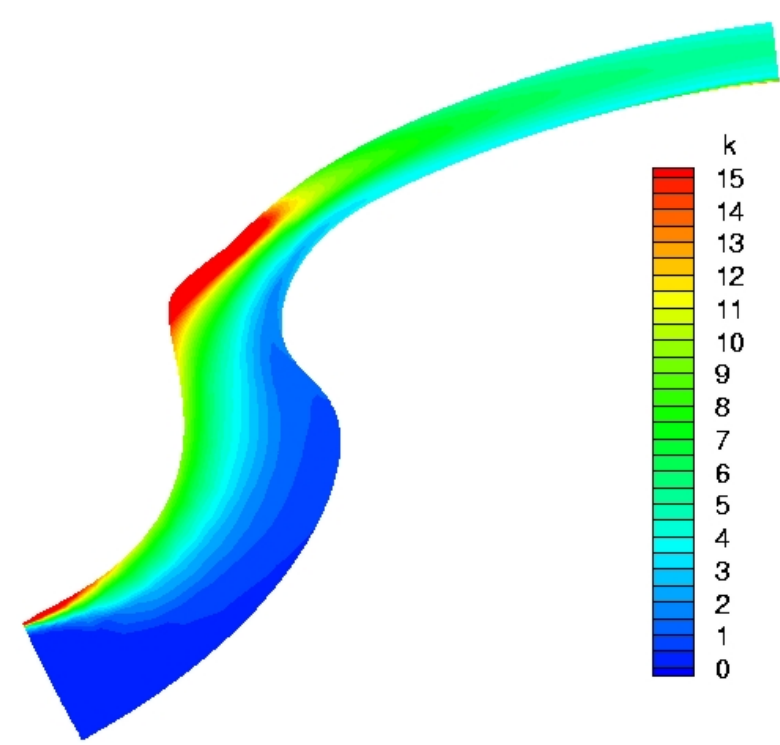

(a) Reference gap target solution

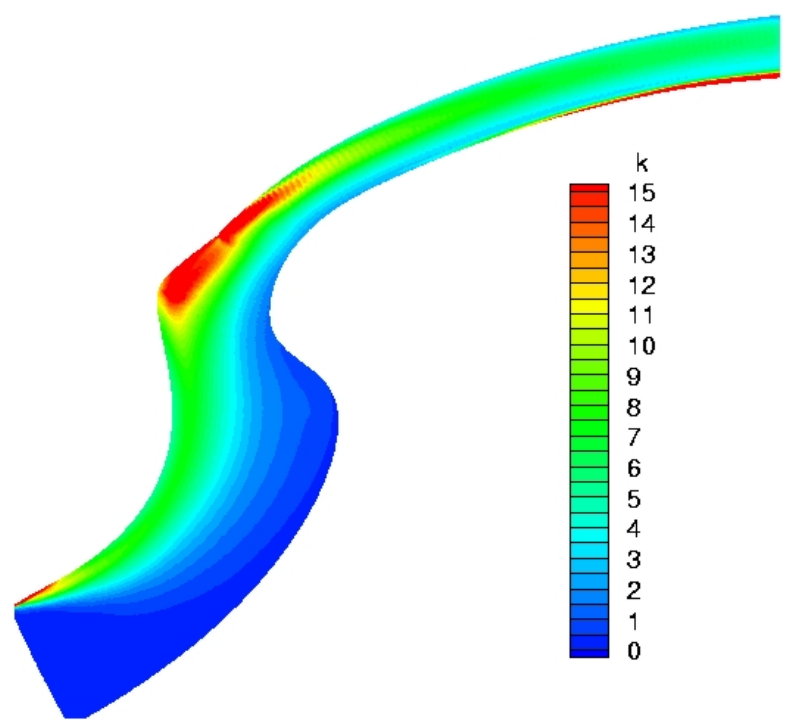

(c) Stochastic realization, 50 streamlines

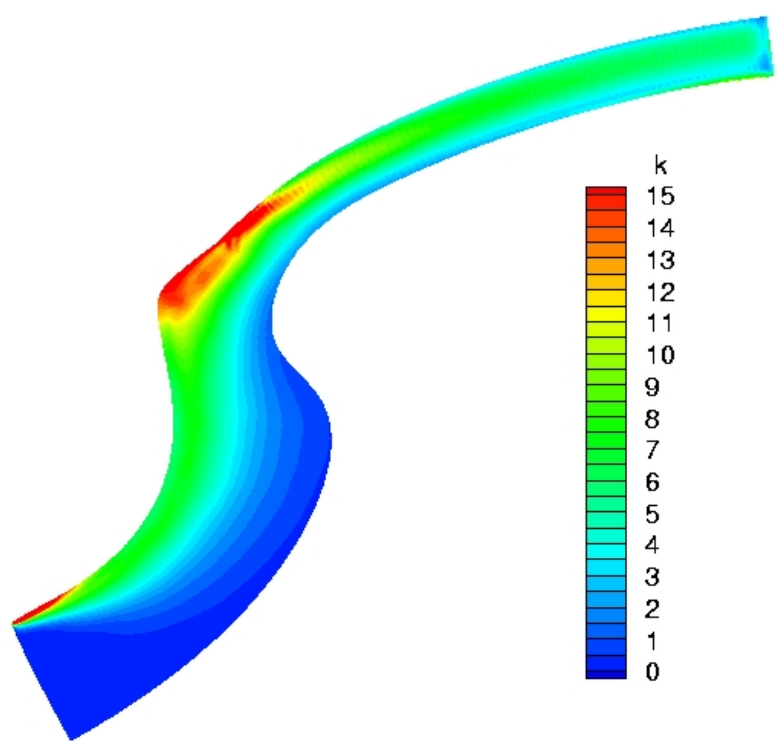

(b) Stochastic realization, 30 streamlines

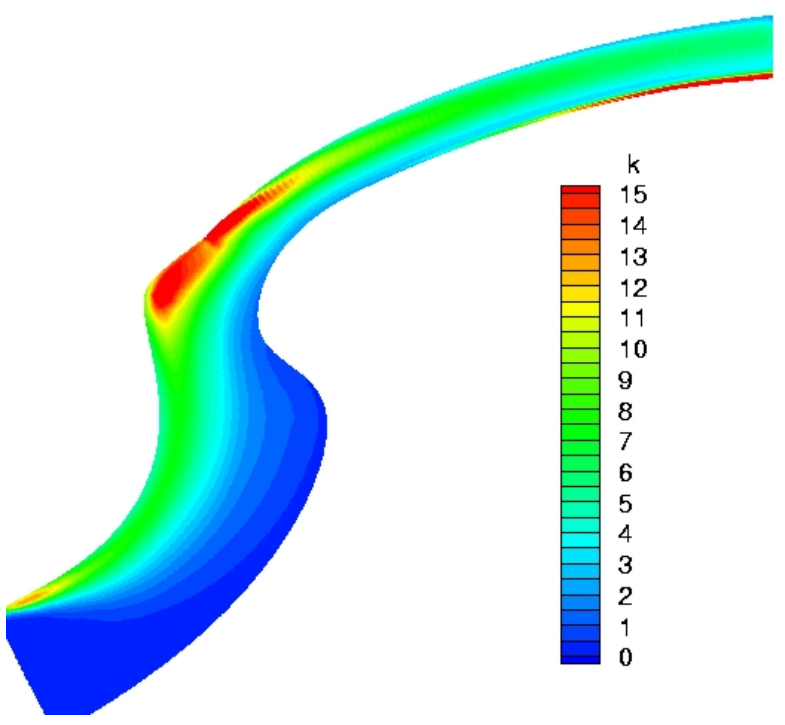

(d) Stochastic realization, 100 streamlines

Figure 14. RANS target distribution of the turbulence kinetic energy on the source patch and results from stochastic realization; reference gap width 


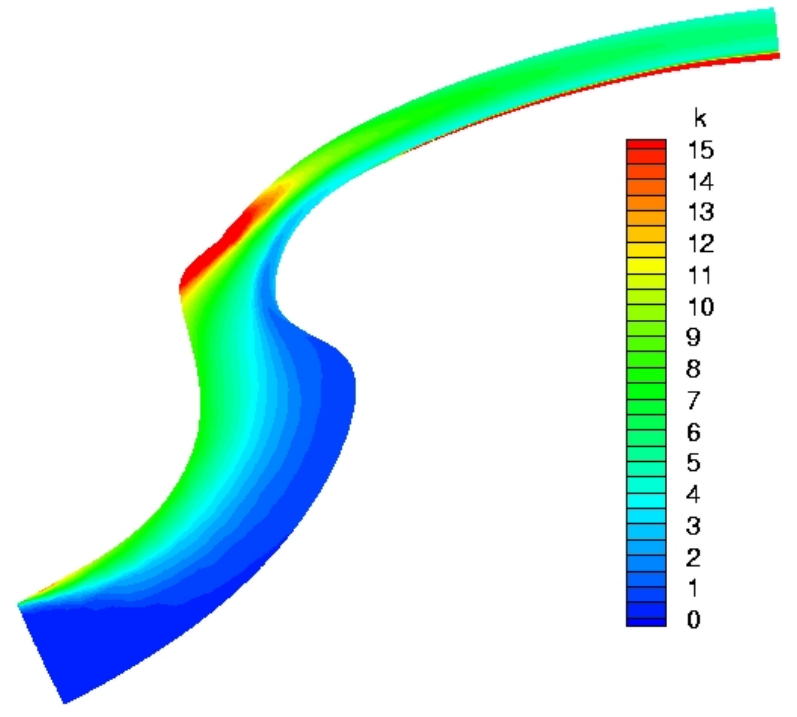

(a) Target solution, small gap width

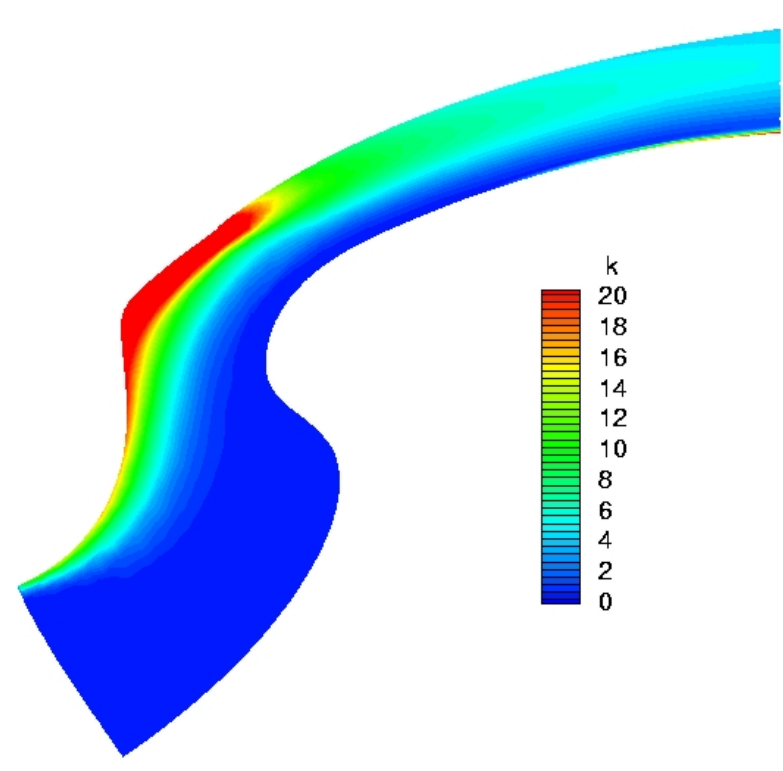

(c) Target solution, large gap width

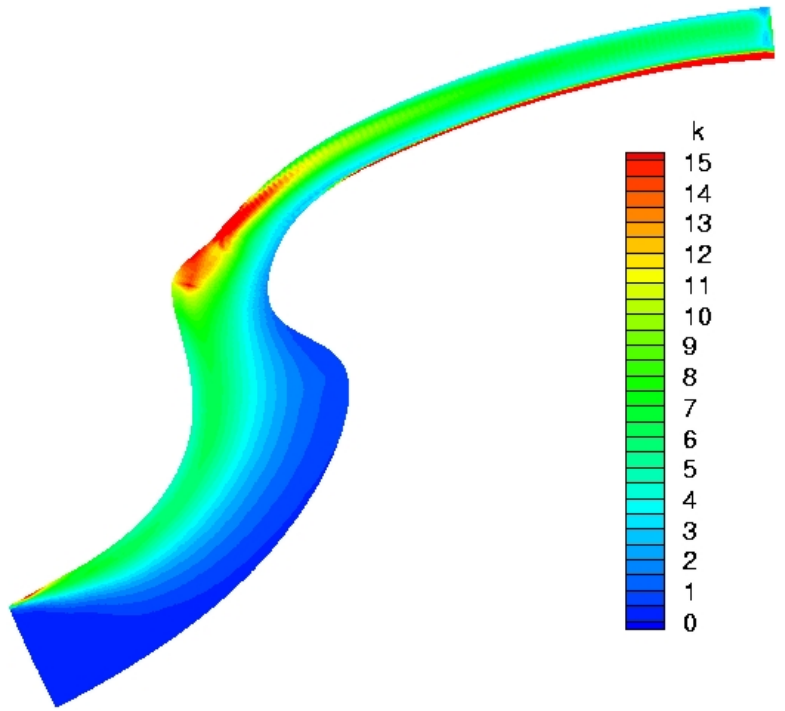

(b) Stochastic realization, small gap width

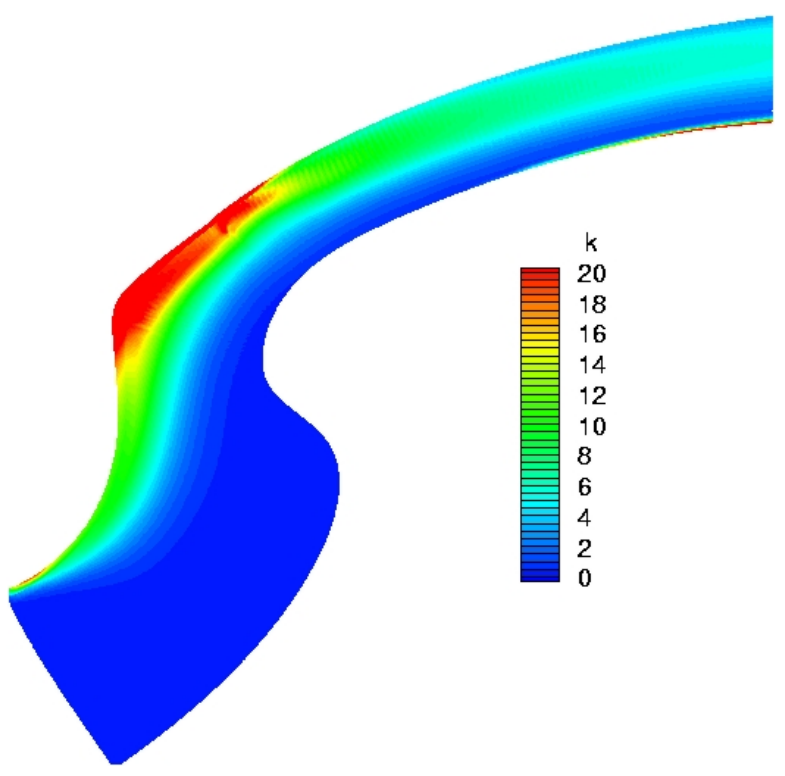

(d) Stochastic realization, large gap

Figure 15. RANS target distributions of the turbulence kinetic energy on the source patch and results from stochastic realization; small and large gap width 


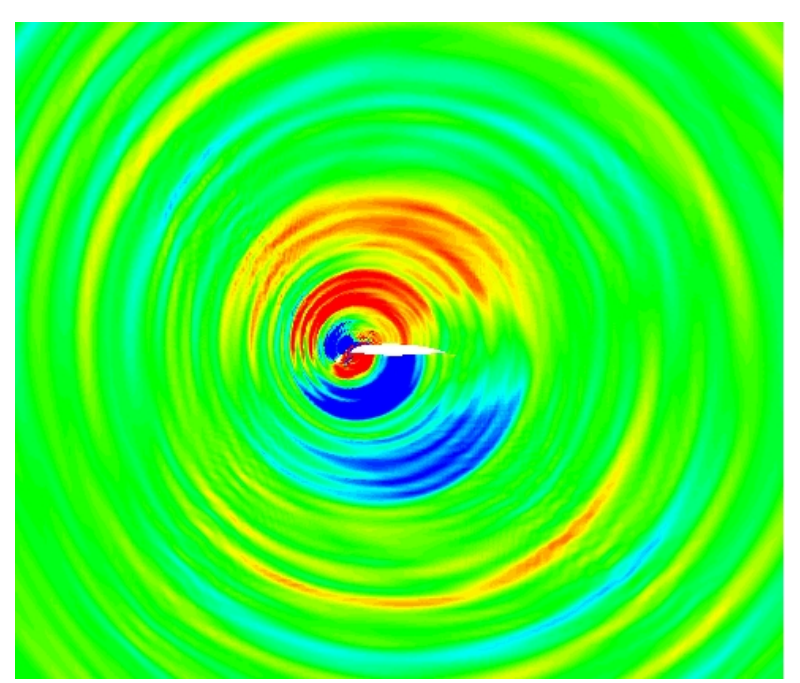

(a) Small slat gap

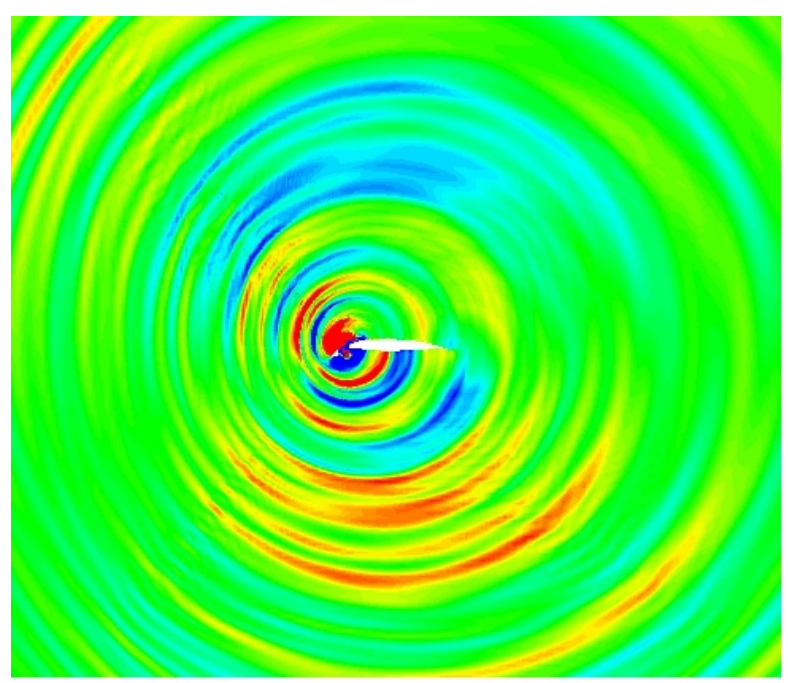

(b) Reference slat gap

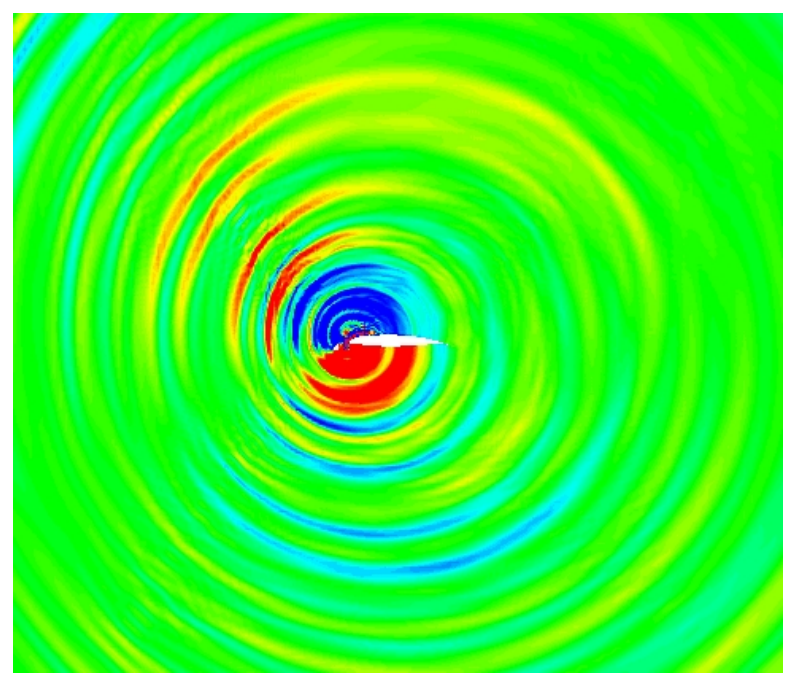

(c) Large slat gap

Figure 16. Instantaneous pressure contours for different slat gap settings (with fixed contour scaling). 


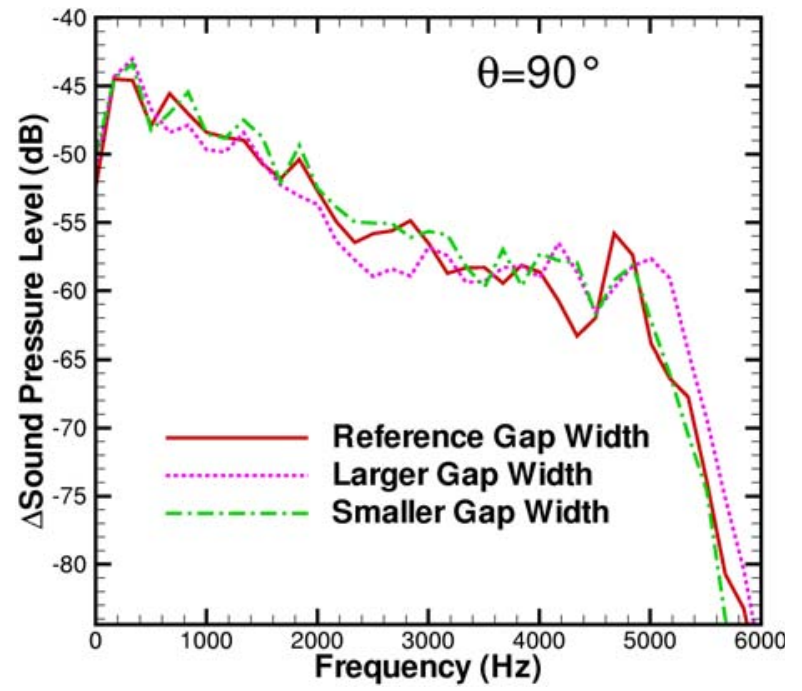

(a) $90^{\circ}$

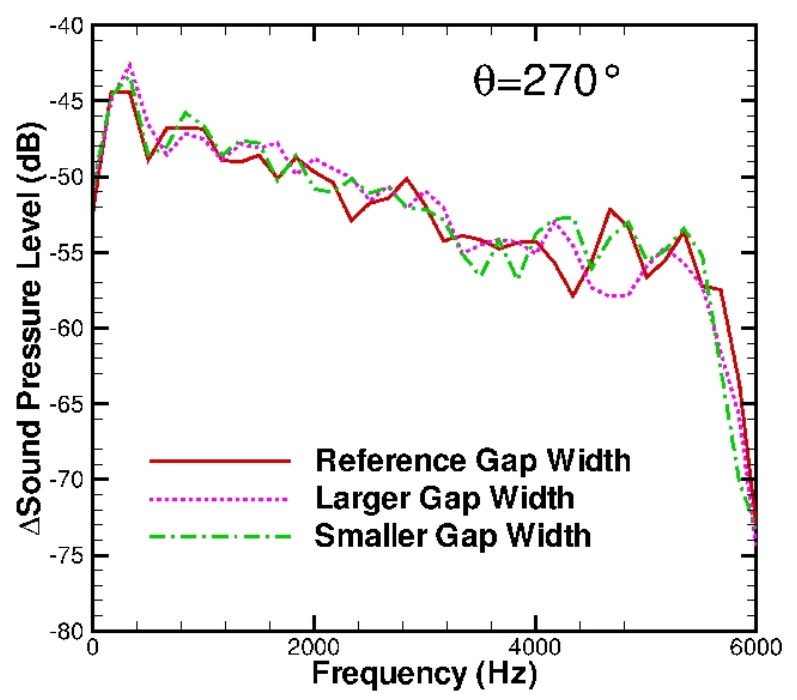

(c) $270^{\circ}$

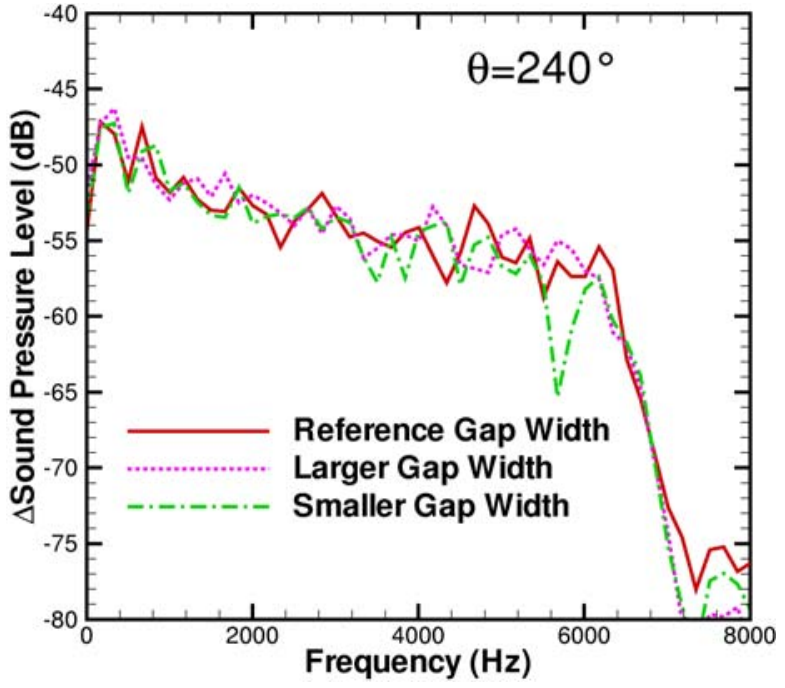

(b) $240^{\circ}$

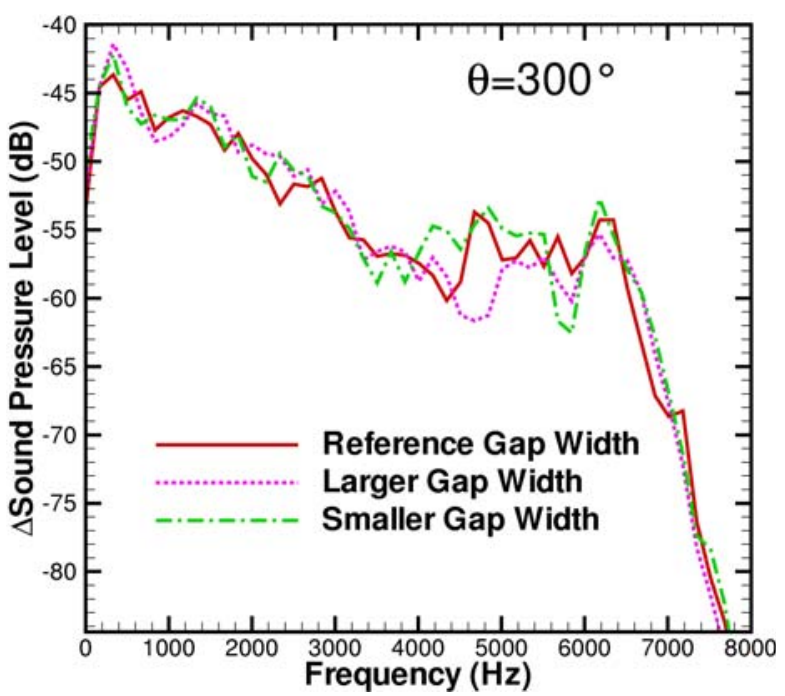

(d) $300^{\circ}$

Figure 17. Narrow band spectra for different polar angles for the low-density grid 


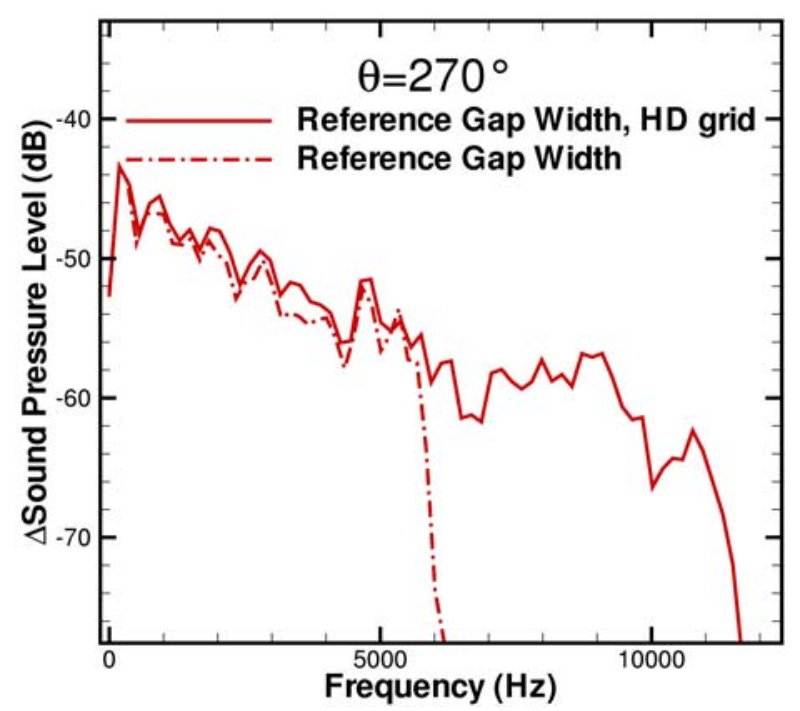

(a) Reference gap width

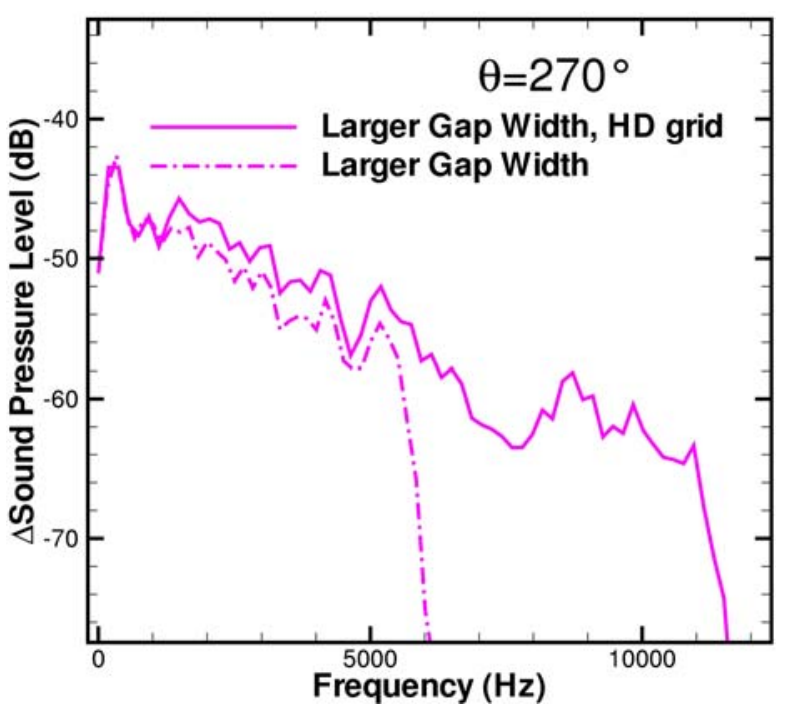

(b) Large gap width

Figure 18. Comparison of narrow band spectra on coarse and refined (HD) grids

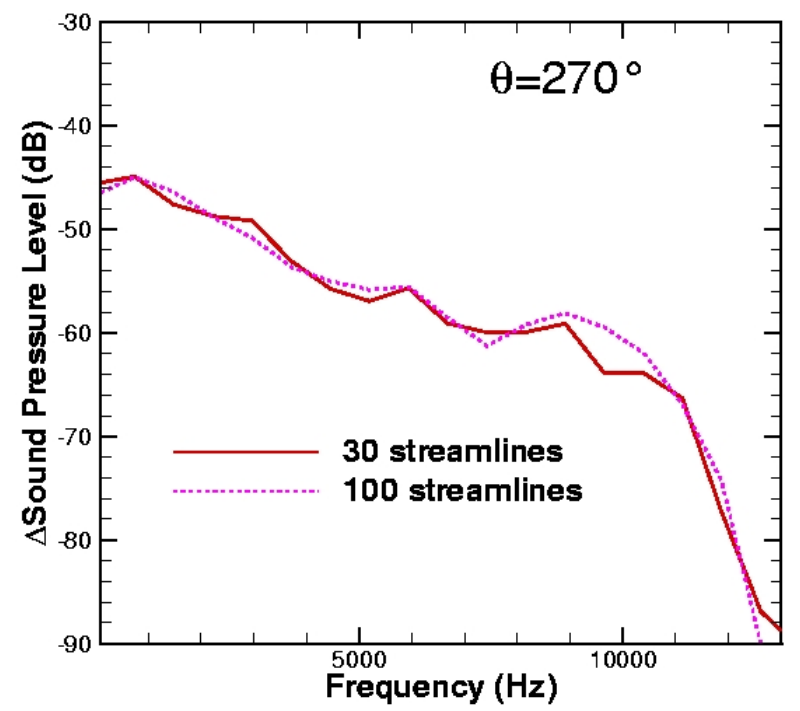

(a) Streamline variation influence on spectrum

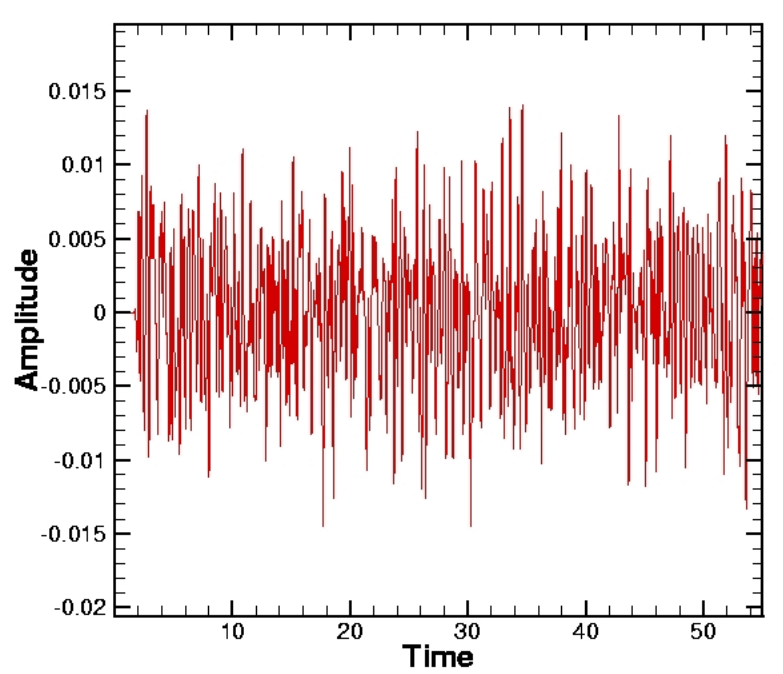

(b) Long time pressure history

Figure 19. Influence of a stochastic model parameter on the predicted acoustic spectrum and long time pressure history over 5e5 CAA time steps 


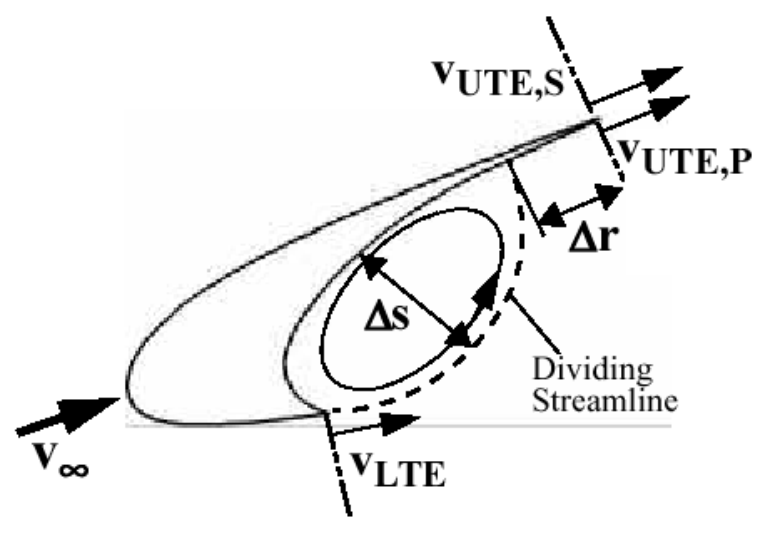

(a) Slat velocity definitions

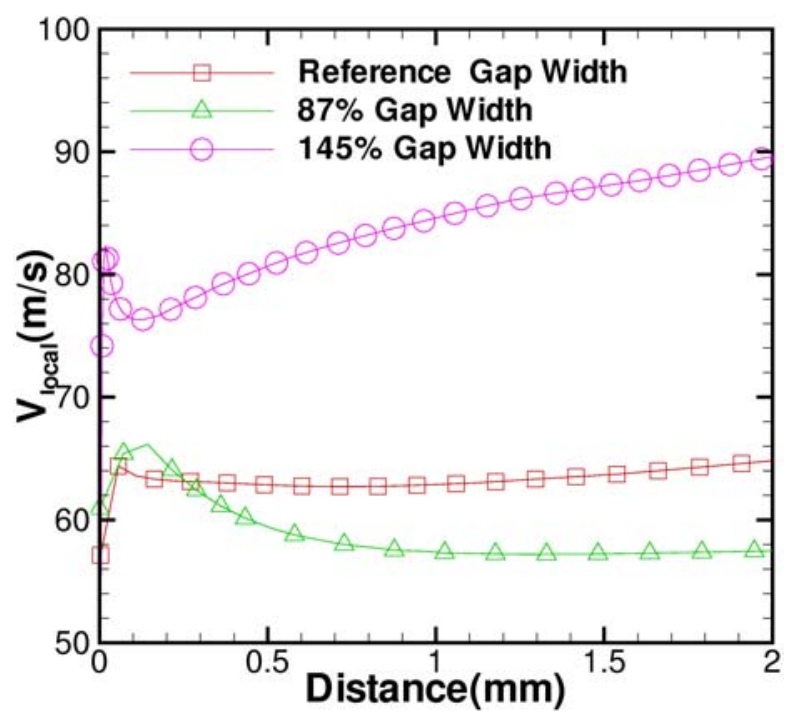

(c) Slat pressure side velocities

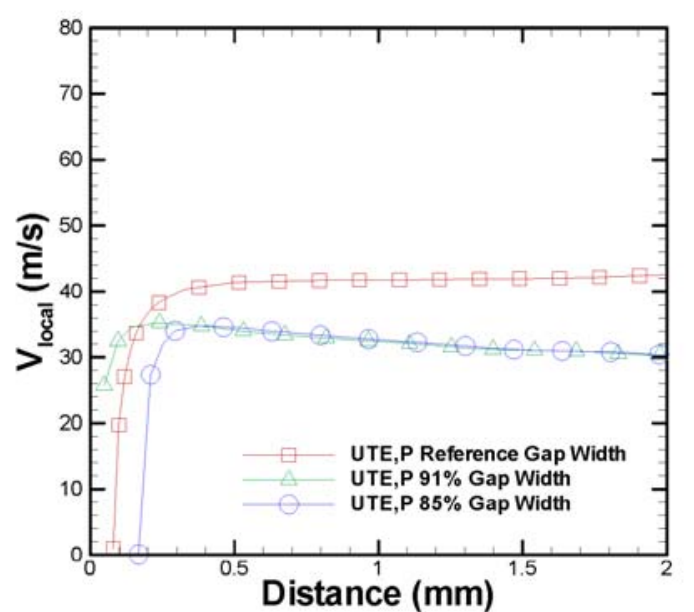

(b) Slat pressure side velocities

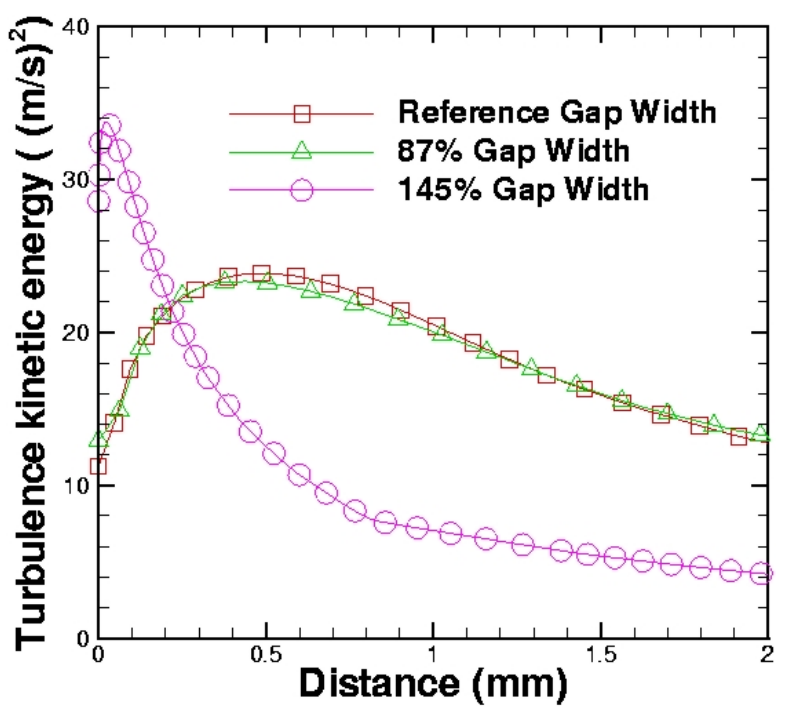

(d) Slat pressure side turbulence energy

Figure 20. RANS solutions normal to the slat pressure side at the trailing edge; comparison of experimental case of Pott-Pollenske et al. ${ }^{25}$ with current two-element configuration 


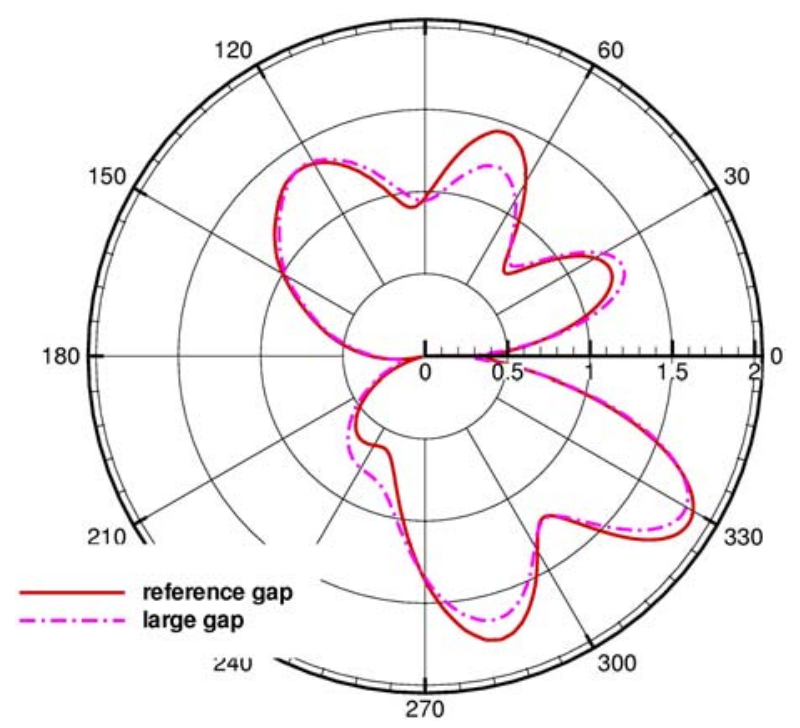

(a) $1 \mathrm{kHz}$

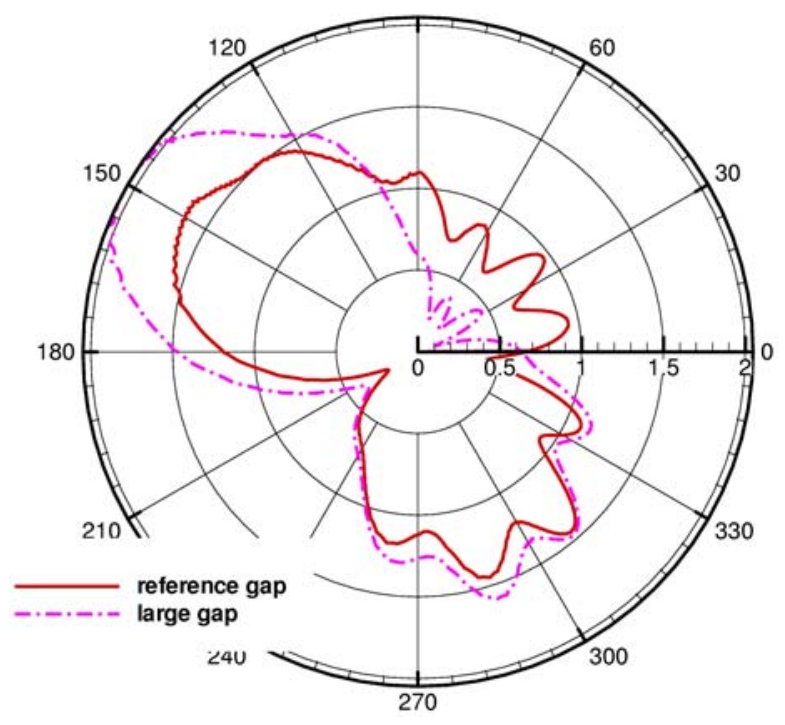

(b) $2 \mathrm{kHz}$

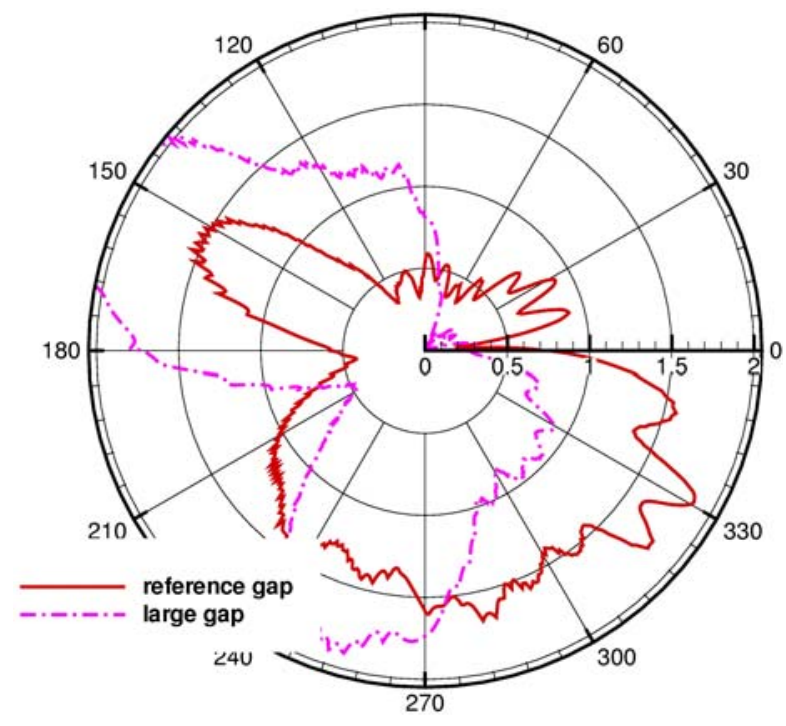

(c) $4 \mathrm{kHz}$

Figure 21. Fine grid directivities 


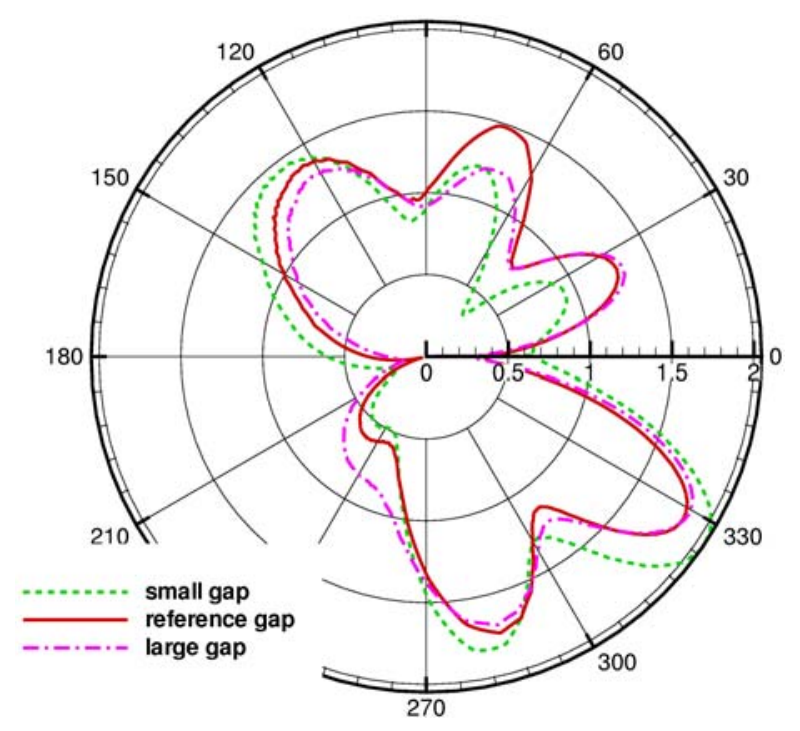

(a) $1 \mathrm{kHz}$

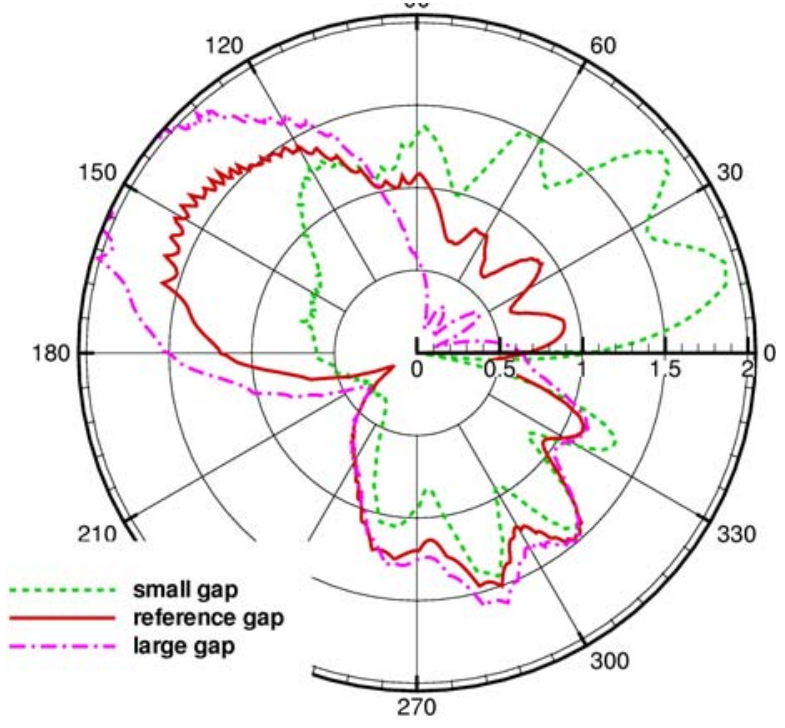

(b) $2 \mathrm{kHz}$

Figure 22. Coarse grid directivities

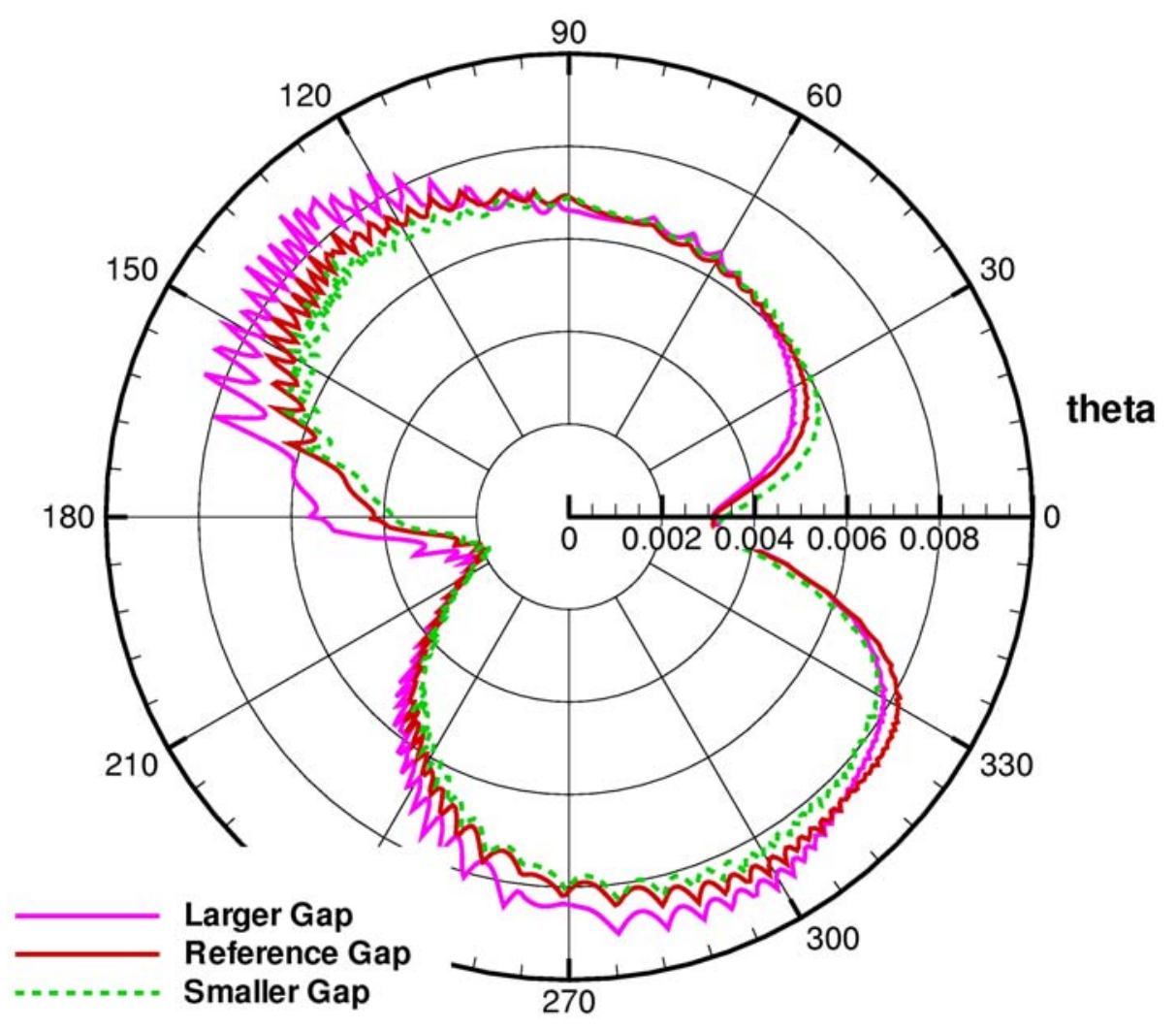

(a)

Figure 23. Root-mean-square (RMŞ3 giregctivity based on coarse grid solution 